\title{
Strategy-proof market clearing mechanisms
}

by

Karol Szwagrzak

Discussion Papers on Business and Economics

No. $4 / 2014$

FURTHER INFORMATION

Department of Business and Economics Faculty of Business and Social Sciences University of Southern Denmark

Campusvej 55

DK-5230 Odense M

Denmark

Tel.: +456550 3271

Fax: +45 65503237

E-mail:1ho@sam.sdu.dk

http://www.sdu.dk/ivoe 


\title{
Strategy-proof market clearing mechanisms*
}

\author{
Karol Szwagrzak ${ }^{\dagger}$
}

March 9, 2014

\begin{abstract}
Consider a market for a resource under disequilibrium prices where suppliers and demanders are privately informed about their optimal supply and consumption levels. Strategy-proof market clearing mechanisms give suppliers and demanders dominant strategy incentives to truthfully reveal this information. We describe the class of strategy-proof and efficient mechanisms responding well to changes in supplies and demands, as formalized by the "replacement principle" (Thomson, 2007). Since no symmetry or anonymity conditions are imposed, these mechanisms can implement a wide array of distributional objectives in both indivisible and divisible resource allocation situations. These mechanisms apply to allocation problems involving network constraints modeling necessary conditions for a transfer of the resource from a supplier to a demander.
\end{abstract}

Keywords: Strategy-proofness; Replacement principle; Network constraints; Indivisible resources JEL classification: D47, D61, D63, C70

\section{Introduction}

We examine a class of allocation problems including a market under disequilibrium prices and the distribution of workloads or support staff within an organization. In

*A previous version was titled "Strategy-proof allocation under network constraints". I am indebted to William Thomson for advice and encouragement that made this paper possible. Vikram Manjunath, Hervé Moulin, and Rodrigo Velez generously offered feedback on previous drafts. I thank Tommy Andersson, Azer Abizade, Paulo Barelli, Albin Erlanson, Jay Sethuraman, and seminar participatnts at Lund University and at the University of Southern Denmark for useful discussions. All errors are my own.

${ }^{\dagger}$ University of Southern Denmark. E-mail: karolszw@sam.sdu.dk 
these situations resources are often allocated through non price-based mechanisms. This paper concerns the design of such mechanisms.

The common feature of the allocation problems studied here is that, barring price adjustments, an allocation simply specifies a number for each individual: a worker receives a certain workload, say 10 hours; a researcher receives a number of hours of research assistant support; a consumer in a price-controlled market receives an amount of the commodity. Moreover, preferences over these assignments display a form of convexity: the closer an assignment is to the optimal one, the better. These preferences are known as single-peaked. ${ }^{1}$

We provide a common framework to study the above mentioned allocation problems and identify mechanisms giving agents dominant strategy incentives to truthfully reveal their preferences. These mechanisms are known as strategy-proof. Our main results, Theorems 1 and 2, describe the class of strategy-proof and efficient mechanisms responding "well" to changes in preferences. This is formalized by a version of the "replacement principle" (Thomson, 2007) roughly specifying that a change in an agent's preferences affects other agents in the same direction welfarewise. Since no symmetry or anonymity conditions are imposed, these mechanisms can implement a wide array of distributional objectives in both indivisible and divisible resource allocation situations.

These mechanisms apply to allocation problmes involving constraints modeled by networks. An example is the assignment of shares of various jobs requiring specific qualifications. The network here connects a worker to the jobs she is qualified for. More generally, this allocation problem can be described as follows:

Unilateral assignment problem Stocks of a resource/workload are to be distributed among a group of agents. Each stock of the resource may be earmarked to be allocated solely among a subgroup of agents. Each agent has single-peaked preferences over the aggregate amount of the resource she receives.

Previous mechanism design work on this problem deals exclusively with perfectly divisible resources. Bochet, Illkılıç, and Moulin (2013) (hereafter, BIM) propose an "egalitarian rule," the only strategy-proof and efficient mechanism recommending allocations satisfying "equal treatment of equals". As with most anonymity or fairness conditions, this one is not meaningful in the presence of the indivisibilities underlying most real-life resource allocation problems. Moreover, considering the diverse

\footnotetext{
${ }^{1}$ In the assignment of workloads under fixed wages, if the worker's disutility of labor is a convex function of labor supplied, then her preferences over workloads will be single-peaked.
} 
situations that feature the structure of the unilateral assignment problem, we are agnostic as to the appropriateness of any one fairness condition. Our objective is to gain a complete understanding of all well behaved strategy-proof and efficient allocation mechanisms. (As it turns out, BIM's egalitarian rule is an example of the mechanisms we propose.)

Our results apply in a two-sided version of the unilateral assignment problem. Instead of there being stocks of the resource to be allocated, there are suppliers of the resource, with preferences over their supplied amounts. The separation of agents into suppliers and demanders has multiple interpretations. Besides the distinction between producers and consumers, one may consider service providers, some beyond and some below their optimal service load. The allocation problem then becomes transferring shares of the service loads from overloaded providers to under-loaded ones. There is also a bilateral time-matching interpretation where the a network connection models compatibility between a pair of agents. ${ }^{2}$

Bilateral assignment problem A resource is to be transferred from its suppliers to its demanders. A supplier can only transfer an amount of the resource to demanders connected to her by a network. Suppliers and demanders have single-peaked preferences over the their supplied and acquired amounts, respectively.

The bilateral assignment problem was introduced by Bochet, İlkılıç, Moulin, and Sethuraman (2012) (hereafter, BIMS). ${ }^{3}$ BIMS also introduce an egalitarian mechanisms for this problem. A defining property of this mechanism is again "equal treatment of equals," incompatible with indivisibilities. (For the special case where resources are divisible, BIMS' egalitarian rule is also an example of the mechanisms we propose.)

The rest of this paper is organized as follows. Section 2 lays out the framework and definitions. Section 3 contains descriptions of the set of feasible and efficient allocations for the bilateral and unilateral assignment problems. Section 4 discusses the "replacement principle." Section 5 describes our class of mechanisms and presents the main results concerning them. Section 6 illustrates the distributional objectives that can be achieved using these mechanisms. Though our analysis in Sections 3 through 6 focuses on bilateral assignment problems, in Section 7 we show how our

\footnotetext{
${ }^{2}$ Bogomolnaia and Moulin (2004) consider the case where suppliers (women) and demanders (men) have dichotomous preferences over each other. A network connection here models a woman and man finding each other mutually acceptable.

${ }^{3}$ Kıbrıs and Küçükşenel (2009) studied a non-networked version of the problem where all suppliers can transfer to all demanders.
} 
results extend to unilateral assignment problems. An Appendix gathers the proofs not included in the body of the paper.

\section{Framework}

A resource, available in either divisible or indivisible units, is to be transferred from a finite group of suppliers/sources $S$ to a finite group of demanders/consumers $D .{ }^{4}$ An agent is any supplier or demander. Let $\boldsymbol{N}$ denote the agent set, $S \cup D$, and $\boldsymbol{n}$ denote its cardinality.

The requirement that a certain supplier can only transfer to certain demanders is modeled by specifying connections in a network. Transfer opportunities are represented by edges in a bipartite graph $\boldsymbol{G}$ linking demanders and suppliers: $j \in D$ can receive from $i \in S$ only if there is an edge, denoted $\boldsymbol{i j}$, in graph $G$. Without loss of generality, we assume that $G$ is connected. ${ }^{5}$ For each agent $i \in N$, let $\Gamma(\boldsymbol{i})$ denote all the agents $j \in N$ that are connected to $i$ in $G$, i.e. such that $i j \in G$. For each $I \subseteq J \subseteq N$, let $\Gamma(I) \equiv \cup_{i \in I} \Gamma(i)$, let $\Gamma(I ; J) \equiv \Gamma(I) \cap J$, and let $G[I]$ denote the sub-graph consisting of the edges in $G$ between agents in $I .{ }^{6}$

Each agent $i$ can receive assignment within a range, modeling capacity constraints. We refer to this range as the agent's assignment space and denote it $A_{\boldsymbol{i}}$. If the resource is available in indivisible units $A_{i}$ is an interval in $\mathbb{Z}_{+} \cdot{ }^{7}$ If the resource is available in divisible units $A_{i}$ is an interval in $\mathbb{R}_{+}$. Let $\boldsymbol{X}_{\boldsymbol{i}}$ denote the upper bound of $A_{i}$. The agent is equipped with a single-peaked preference relation $\boldsymbol{R}_{\boldsymbol{i}}$ over her assignment space: there is a number $\boldsymbol{p}\left(\boldsymbol{R}_{\boldsymbol{i}}\right)$ in $A_{i}$ such that for each pair $x_{i}, y_{i}$ in $A_{i}$, if $x_{i}<y_{i} \leq p\left(R_{i}\right)$ or $p\left(R_{i}\right) \leq y_{i}<x_{i}$, then $y_{i} P_{i} x_{i}{ }^{8}$ We refer to $p\left(R_{i}\right)$ as the peak of $\boldsymbol{R}_{\boldsymbol{i}}$, or simply as agent $\boldsymbol{i}$ 's peak when there is no room for confusion. Let $\boldsymbol{R}_{\boldsymbol{i}}$ denote this class of these preferences. Let $\boldsymbol{R} \equiv\left(R_{i}\right)_{i \in N}$ and $\boldsymbol{p}(\boldsymbol{R}) \equiv\left(p\left(R_{i}\right)\right)_{i \in N}$.

Feasible allocations A feasible allocation is a list $x \equiv\left(x_{i}\right)_{i \in N} \in A^{N}$ specifying the assignments for each agent; these assignments are such that there is a matrix $\left(x_{i j}\right)$

\footnotetext{
${ }^{4}$ The basic mathematical notation is as follows: let $\left\{Y_{i}\right\}_{i \in I}$ be a family of sets $Y_{i}$ indexed by $I$. Let $Y^{I} \equiv \times_{i \in I} Y_{i}$. For each $y \in Y^{I}$ and each $J \subseteq I$, we denote by $y_{J}$ the projection of $y$ onto $Y^{J}$. If $x, y \in \mathbb{R}^{I}$, then $x \geq y$ means that, for each $i \in I, x_{i} \geq y_{i}$.

${ }^{5}$ Formally, $G$ is connected if there is a path between any two agents: for each pair $i, j \in N$, there are $k_{1}, k_{2}, \ldots, k_{l} \in N$ such that $i k_{1}, \ldots, k_{l} j \in G$. To see why this assumption is without loss of generality, note that if it were not met then $G$ could be partitioned into disjoint subgraphs, each connected and indpendent. We could then study each of this subgraphs separately.

${ }^{6}$ Graph $G[I]$ is known as the subgraph induced by the nodes in $I$.

${ }^{7}$ Here, an interval is the set $\{l, l+1, \ldots, l+k\}$ for non-negative integers $l$ and $k$.

${ }^{8}$ As usual $P_{i}$ denotes the asymmetric part of $R_{i}$.
} 
of non-negative numbers satisfying, for each $(i, j) \in S \times D$, (i) $x_{i j}>0$ only if $i j \in G$, (ii) $x_{j}=\sum_{i \in S} x_{i j}$, and (iii) $x_{i}=\sum_{j \in D} x_{i j}$. If the resource comes in indivisible units, the entries $x_{i j}$ are integers. A matrix $\left(x_{\boldsymbol{i}}\right)$ implements allocation $x$ if it satisfies (i)-(iii). Let $Z$ denote the set of feasible allocations. Similarly, for each $I \subseteq N$, let $\boldsymbol{Z}(\boldsymbol{I})$ denote the allocations that can be implemented solely within the agents in $I{ }^{9}$

Mechanisms A mechanism $\varphi$ is a function that recommends, for each preference profile $R \in \mathcal{R}^{N}$, a unique feasible allocation denoted $\varphi(R)$. For each $R \in \mathcal{R}^{N}$, let $P(R)$ denote the set of (Pareto) efficient allocations at $R \cdot{ }^{10}$ A mechanism $\varphi$ is efficient if it only recommends efficient allocations: for each $R \in \mathcal{R}^{N}, \varphi(R) \in P(R)$. The basic incentive compatibility criterion studied in this paper is strategy-proofness, the requirement that reporting preferences truthfully is a dominant strategy for each agent. A mechanism $\varphi$ is strategy-proof if, for each $R \in \mathcal{R}^{N}$, each $i \in N$, and each $R_{i}^{\prime} \in \mathcal{R}_{i}, \varphi_{i}(R) R_{i} \varphi_{i}\left(R_{i}^{\prime}, R_{-i}\right)$.

Special cases The bilateral and unilateral assignment problems discussed in the Introduction are embedded in this framework as follows:

Bilateral assignment For each agent $i, A_{i}$ is $\left[0, X_{i}\right]$ if the resource is divisible and $A_{i}$ is $\left\{0,1, \ldots, X_{i}\right\}$ if the resource is available in indivisible units.

Unilateral assignment For each demander $i, A_{i}$ is a singleton and, for each supplier $i, A_{i}$ is $\left[0, X_{i}\right]$ if the resource is divisible and $A_{i}$ is $\left\{0,1, \ldots, X_{i}\right\}$ if the resource is available in indivisible units.

Though our analysis in Sections 3 through 6 focuses on bilateral assignment problems, in Section 7 we show how our results extend to unilateral assignment problems.

A basic instance of the unilateral assignment problem was introduced by Sprumont (1991). Here, a single stock of an infinitely divisible resource is to be distributed among a group agents whose preferences over assignments are single-peaked.

Sprumont's model There is a single demander $i$, her assignment space $A_{i}$ is $\left\{X_{i}\right\}$, and the graph $G$ connects all suppliers to demander $i$, $G=\{j i: j \in S\}$. For each supplier $j, X_{j}=X_{i}$ and $A_{j}=\left[0, X_{i}\right]$.

\footnotetext{
${ }^{9}$ That is, $x \in Z(I)$ if $x \in A^{I}$ and there is a matrix $\left(x_{i j}: i \in S \cap I, j \in D \cap I\right)$ such that (i) $x_{i j}>0$ only if $i j \in G[I]$, (ii) $x_{j}=\sum_{i \in S \cap I} x_{i j}$, and (iii) $x_{i}=\sum_{j \in D \cap I} x_{i j}$.

${ }^{10}$ An allocation $x \in Z$ is (Pareto) efficient at $R \in \mathcal{R}^{N}$ if there is no $x^{\prime} \in Z$ such that for each $i \in N, x_{i}^{\prime} R_{i} x_{i}$ and, for at least one $i \in N, x_{i}^{\prime} P_{i} x_{i}$.
} 


\section{$3 \quad$ Feasibility and efficiency}

We now present two lemmas used in the analysis of bilateral assignment problems. These lemmas provide polyhedral descriptions of the the set of feasible and efficient allocations, respectively.

A version of the "Supply-Demand Theorem" (Gale, 1957) yields the following description of the set allocations that are feasible within each group of agents.

Lemma 1 (Feasibility). For each $I \subseteq N, x \in Z(I)$ is equivalent to either of the following statements:

(a) for each $J \subseteq S \cap I, \sum_{J} x_{i} \leq \min \left\{\sum_{\Gamma(K ; I)} x_{i}+\sum_{J \backslash K} X_{i}: K \subseteq J\right\}$, and $\sum_{S} x_{i}=\sum_{D} x_{i}$

(b) for each $J \subseteq D \cap I, \sum_{J} x_{i} \leq \min \left\{\sum_{\Gamma(K ; I)} x_{i}+\sum_{J \backslash K} X_{i}: K \subseteq J\right\}$, and $\sum_{S} x_{i}=\sum_{D} x_{i}$

We now introduce the key element in the description of the set of efficient allocations, a version of the Gallai-Edmonds decomposition of bipartite graph. For each $R \in \mathcal{R}^{N}$, we define the imbalance between the supply of a group of suppliers $I \subseteq S$ and the demands of demanders connected to them:

$$
f(I) \equiv \sum_{I} p\left(R_{i}\right)-\sum_{\Gamma(I)} p\left(R_{i}\right)
$$

Because $f: 2^{S} \rightarrow \mathbb{R}$ is super-modular, ${ }^{11}$ the class of subsets of $S$ maximizing $f$ is closed under unions and intersections. Thus, there is a unique inclusion-minimal subset of $S$ maximizing $f$. Let $S_{-}$denote it if there is $I \subseteq S$ with $f(I)>0$ and let $S_{+}$denote its complement in $S, S \backslash S_{-}$. Otherwise let $S_{+}=S$. Thus, at most one of $S_{-}$and $S_{+}$is empty. Let $\boldsymbol{D}_{+}$denote the demanders connected to suppliers in $S_{-}$, that is $D_{+}=\Gamma\left(S_{-}\right)$, and let $D_{-}$denote $D \backslash D_{+}$. The sets $S_{-}, S_{+}, D_{-}, D_{+}$partition $N$. This partition is the Gallai-Edmonds decomposition corresponding to preference profile $R$. For each $R \in \mathcal{R}^{N}$, let $\mathbb{P}(R)$ denote the partition of $N$ derived from $R$ in this way. This partition is important in describing the set of efficient allocations.

Lemma 2 (Efficiency). Let $R \in \mathcal{R}^{N}$ and let $S_{-}, S_{+}, D_{-}, D_{+}$denote the cells of partition $\mathbb{P}(R)$. Then $x \in P(R)$ if and only if

(a) $x_{S_{-} \cup D_{+}} \in Z\left(S_{-} \cup D_{+}\right), x_{S_{+} \cup D_{-}} \in Z\left(S_{+} \cup D_{-}\right)$;

(b) for each $i \in S_{-} \cup D_{-}, x_{i} \leq p\left(R_{i}\right)$; for each $i \in S_{+} \cup D_{+}, x_{i} \geq p\left(R_{i}\right)$.

\footnotetext{
${ }^{11}$ For each pair $I, J \subseteq S, f(I)+f(J) \leq f(I \cup J)+f(I \cap J)$.
} 
The above Lemma generalizes BIMS description of the efficient set of a bilateral assignment problem (Proposition 1 in BIMS) to situations featuring indivisibilities. In fact, non of the arguments in Proposition 1 of BIMS relies on the divisibility of resources. ${ }^{12}$

\section{The replacement principle}

There are strategy-proof and efficient mechanisms with a number of undesirable features: their recommended allocations change dramatically in response to small preference variations; their informational requirements are taxing and they are highly bossy in the sense of Satterthwaite and Sonnenschein (1981). These flaws are due to their unstructured response to preference changes. The task addressed here is that of specifying how mechanisms should respond to preference changes.

We build on intuition from a workload allocation problem. When an employee expresses a greater willingness to work, it is reasonable that no other workers are forced to work more as a result. This is an expression of the "replacement principle" in the axiomatic theory of resource allocation. ${ }^{13}$ The principle asserts that a change or "replacement" in an agent's preferences ought to affect all other agents in the same way welfare-wise, they are all at least as well off or they are all at most as well off as before the change. As formulated by Thomson (1997), the condition is:

Welfare-dominance under preference-replacement (WDUPR): For each $R \in \mathcal{R}^{N}$, each $i \in N$, and each $R_{i}^{\prime} \in \mathcal{R}_{i}$, either [for each $j \in N \backslash\{i\}, \varphi_{j}(R) R_{j}$ $\varphi\left(R_{i}^{\prime}, R_{-i}\right)$ ] or [for each $\left.j \in N \backslash\{i\}, \varphi_{j}\left(R_{i}^{\prime}, R_{-i}\right) R_{j} \varphi_{j}(R)\right]$.

Even in Sprumont's basic model (see Section 2) WDUPR is incompatible with basic equity properties (Thomson, 1997) and intuitive strategy-proof mechanisms such as sequential dictatorships do not satisfy it. However, the examples illustrating these incompatibilities are somewhat artificial: the change in an agent's preferences has to be large enough to take an economy were there is too little to distribute to one were there is too much, or conversely. In large scale resource allocation problems this is unlikely to be realistic. Qualifying WDUPR so that it will hold in situations were the preference changes are not this disruptive yields a requirement

\footnotetext{
${ }^{12}$ The background network flow tools used to establish Proposition 1 of BIMS also apply to the case of indivisibilities. See, for instance Corollary 8.7 in Korte and Vygen (2001).

${ }^{13}$ See Thomson $(2007,1999)$ for an overview of the literature on the replacement principle. The earliest instance of the property is "agreement" in (Moulin, 1987).
} 
that is fully compatible with efficiency and various equity notions. This qualified version is satisfied by many intuitively appealing mechanisms in Sprumont's model (Thomson, 1997).

The challenge is thus to qualify WDUPR, adapting it to the networked environments studied here. A straightforward adaptation of Thomson's qualified WDUPR in a bilateral assignment problem, where every supplier is connected to every demander, is to require that WDUPR holds as long as the change in an agent's preferences does not take an economy where the sum of the demanders' preferred transfers is greater than the sum of suppliers preferred transfers' to one where the opposite is true. The key then is in formalizing how a change in preferences affects overall scarcity under general network constraints.

The Gallai-Edmonds decomposition derived in Section 3 enables us to canonically distinguish a pattern of scarcity or abundance in the relationship between the demands of some agents and the supplies available to them, for any network. For each preference profile $R$, the corresponding partition of agents into $S_{-}, S_{+}, D_{+}$, and $D_{-}$ derived in Section 3 is this Gallai-Edmonds decomposition. Each group of demanders in $D_{+}$is "over-supplied" by the suppliers in $S_{-}$who can only supply to them because $\Gamma\left(S_{-}\right)=D_{+}$. Similarly, each group of suppliers in $S_{+}$is "over-demanded" by the demanders in $D_{-}$who can only receive the resource from them because $\Gamma\left(D_{-}\right)=S_{+}$.

We will require WDUPR to hold for changes in preferences that do not alter the configuration of over- and under-supply in a networked economy, as formalized by the Gallai-Edmonds decomposition:

Replacement-dominance: For each $R \in \mathcal{R}^{N}$, each $i \in N$, and each $R_{i}^{\prime} \in \mathcal{R}_{i}$, if $\mathbb{P}(R)=\mathbb{P}\left(R_{i}^{\prime}, R_{-i}\right)$ then, either [for each $j \in N \backslash\{i\}, \varphi_{j}(R) R_{j} \varphi\left(R_{i}^{\prime}, R_{-i}\right)$ ] or [for each $\left.j \in N \backslash\{i\}, \varphi_{j}\left(R_{i}^{\prime}, R_{-i}\right) R_{j} \varphi_{j}(R)\right]$.

In Sprumont's model, replacement-dominance coincides with Thomson's qualified WDUPR. Under efficiency, it also coincides with "replacement monotonicity" (Barberà et al., 1997). This is the requirement that if an agent's preferences change leading to an increase in her assignment, then all other agents receive at most as much as they did before. This is a restriction on physical assignments and not on welfare. Beyond Sprumont's model, replacement monotonicity can be defined as follows:

Replacement-monotonicity: For each $R \in \mathcal{R}^{N}$, each $i \in S$, and each $R_{i}^{\prime} \in \mathcal{R}_{i}$, $\varphi_{i}\left(R_{i}^{\prime}, R_{-i}\right) \geq \varphi_{i}(R)$ implies [for each $j \in S \backslash\{i\}, \varphi_{j}\left(R_{i}^{\prime}, R_{-i}\right) \leq \varphi_{j}(R)$ ] and [for each $\left.j \in D, \varphi_{j}\left(R_{i}^{\prime}, R_{-i}\right) \geq \varphi_{j}(R)\right]$. The statement holds when the roles of $S$ and $D$ are 
reversed.

Replacement-monotonicity is stronger than replacement-dominance. As we show in Lemma 5 (see Appendix C), a strategy-proof, efficient, and replacement-dominant mechanism satisfies a weak version of replacement-monotonicity.

\section{Adjustment mechanisms}

The mechanisms proposed here are described by means of an adjustment process starting from a set of initial allocations. These allocations can be interpreted as providing welfare guarantees: after the adjustment process has ended, each agent is at least as well off as if she had kept her initial assignment.

Intuitively, an adjustment mechanism operates as follows. For all preference profile $R$ inducing the same Gallai-Edmonds decomposition, the adjustment function specifies the same initial allocation, say $q^{0}$. For each agent $i$, her initial assignment-her component of $q^{0}$ - defines the endpoint of an interval from where she is free to choose her preferred assignment. Depending on $i$ 's location in the network this interval will be of the form

$$
\left[0, q_{i}^{0}\right] \text { or }\left[q_{i}^{0}, X_{i}\right]
$$

Suppose $i$ is a demander choosing from $\left[0, q_{i}^{0}\right]$ and her peak, $p_{i}$, is smaller than $q_{i}^{0}$. Then $i$ receives $p_{i}$ and "frees" an amount $q_{i}^{0}-p_{i}$ of the resource. This excess will be redistributed among the remaining agents whose intervals did not enable them to obtain their preferred consumption level. For instance, if there is $j \in D$ such that $p_{j} \notin\left[0, q_{j}^{0}\right]$ or $j \in S$ such that $p_{j} \notin\left[q_{j}^{0}, X_{j}\right]$, then, upon the release of the excess resources from the agents who were able to obtain their peak assignments, the endpoint of $j$ 's interval is adjusted to $q_{j}^{1}$ so that, respectively,

$$
\left[0, q_{j}^{1}\right] \supseteq\left[0, q_{j}^{0}\right] \quad \text { or } \quad\left[q_{j}^{1}, X_{j}\right] \supseteq\left[q_{j}^{0}, X_{j}\right] .
$$

Note that such adjustment will occur only if there is an agent whose peak did lie in her interval. For such an agent, the adjustment will then yield an assignment equal to her peak. Thus, by construction, there will be at most as many adjustments as there are agents, $n$.

\subsection{Definition}

For each $p \in A^{N}$, we say that $p$ induces $C$ if there is a preference profile $R$ such that $p=p(R), \mathbb{P}(R)=\left\{S_{-}, S_{+}, D_{-}, D_{+}\right\}$, and $C=S_{-} \cup D_{+}$. Let $\mathcal{C}$ denote the collection 
of all $C \subseteq N$ for which there is a $p$ in $A^{N}$ such that $p$ induces $C$. For each $C \in \mathcal{C}$, let $\boldsymbol{q}^{\boldsymbol{C}}$ denote an "initial" allocation in $Z(C) \times Z(N \backslash C)$.

An adjustment function $g: Z \times A^{N} \rightarrow Z$ is a function such that,

if $p \in A^{N}$ induces $C$, and $q^{1} \equiv g\left(q^{C}, p\right), q^{2} \equiv g\left(q^{1}, p\right), \ldots, q^{n} \equiv g\left(q^{n-1}, p\right)$

then, for each $t \in\{1, \ldots, n\}, q^{t} \in Z(C) \times Z(N \backslash C)$ and

(a) $\quad q_{i}^{t}=p_{i} \quad$ if $i \in[C \cap S] \cup[D \backslash C]$ and $\quad q_{i}^{t-1} \geq p_{i}$

(b) $\quad$ or $i \notin[C \cap S] \cup[D \backslash C]$ and $\quad q_{i}^{t-1} \leq p_{i}$

(b) $\quad q_{i}^{t} \geq q_{i}^{t-1} \quad$ if $i \in[C \cap S] \cup[D \backslash C]$ and $\quad q_{i}^{t-1}<p_{i}$

$q_{i}^{t} \leq q_{i}^{t-1} \quad$ if $\quad i \notin[C \cap S] \cup[D \backslash C]$ and $\quad q_{i}^{t-1}>p_{i}$

(c) $q^{t}=g\left(q^{t-1}, \tilde{p}_{i}, p_{-i}\right) \quad$ if $\quad i \in[C \cap S] \cup[D \backslash C]$ and $\quad \tilde{p}_{i} \geq p_{i}>q_{i}^{t-1}$

or $i \notin[C \cap S] \cup[D \backslash C]$ and $\quad \tilde{p}_{i} \leq p_{i}<q_{i}^{t-1}$.

Let $\mathcal{H}$ consist of all adjustment functions. Each adjustment function $g \in \mathcal{H}$ specifies a unique mechanism we denote $\boldsymbol{\varphi}^{\boldsymbol{g}}$. We refer to them as adjustment mechanisms. The allocations recommended by $\varphi^{g}$ are computed as follows: for each $R \in \mathcal{R}^{N}$, if $p(R)$ induces $C$,

$$
\varphi^{g}(R)=\left(q_{i}^{n}\right)_{i \in N}
$$

where, for each $t \in\{1, \ldots, n\}, q^{t} \equiv g\left(q^{t-1}, p(R)\right)$, where $q^{0} \equiv q^{C}$.

In Sprumont's model, adjustment mechanisms are closely related to the mechanisms introduced by Barberà et al. (1997) and Massó and Neme (2007). Examples of adjustment mechanisms can be found in Section 6 .

\subsection{Main results}

Theorem 1. Every strategy-proof, efficient, and replacement-dominant mechanism is an adjustment mechanism.

Strategy-proofness, efficiency, and replacement dominance are thus sufficient conditions for a mechanism to belong to the class of adjustment mechanisms. That is, if $\varphi$ is a mechanism satisfying these properties, then there is an adjustment function

$g \in \mathcal{H}$ such that $\varphi=\varphi^{g}$. Additionally, every adjustment mechanism is strategy-proof and efficient.

Proposition 1. If $g \in \mathcal{H}$, then $\varphi^{g}$ is strategy-proof and efficient. 
By Lemma 5 (in Appendix C), a strategy-proof, efficient and replacement-dominant mechanism satisfies a weak version replacement-monotonicity: in the range of preferences for which the hypothesis of replacement-dominance is satisfied, the mechanism will be replacement-monotonic. Thus, the next corollary follows from Theorem 1 and Lemma 5 (in Appendix C).

Corollary 1. Every strategy-proof, efficient, and replacement-monotonic mechanism is an adjustment mechanism.

\subsubsection{Welfare guarantees}

We identify adjustment mechanisms achieving a wide range of welfare guarantees. These guarantees specify lower bounds on the welfare attainable by each agent. In fact, for any feasible allocation, we can find an adjustment mechanism that makes assignments that each agent finds at least as desirable as receiving her component of the allocation.

We now formalize the requirements on $g \in \mathcal{G}$ that will ensure this. Let $z \in Z$ be the allocation chosen as a welfare guarantee. Let $\left(z_{i j}\right)$ denote a matrix implementing $z$. For each $C$ in $\mathcal{C}$, let $\left(z_{i j}[C]\right)$ denote the matrix obtained form $\left(z_{i j}\right)$ as follows:

$$
z_{i j}[C] \equiv\left\{\begin{array}{cc}
z_{i j} & \text { if } \quad\{i, j\} \subseteq C \text { or }\{i, j\} \subseteq N \backslash C \\
0 & \text { otherwise }
\end{array}\right.
$$

Let $z[C]$ denote the allocation implemented by matrix $\left(z_{i j}[C]\right)$. Note that, by definition, $\left.z[C]\right|_{C} \in Z(C)$ and $\left.z[C]\right|_{N \backslash C} \in Z(N \backslash C)$.

Proposition 2. Let $z \in Z$ and $g \in \mathcal{H}$ be such that

$$
\text { for each } C \in \mathcal{C}, \quad q^{C}=z[C] \text {. }
$$

Then, for each $R \in \mathcal{R}^{N}$ and each $i \in N, \varphi_{i}^{g}(R) R_{i} z_{i}$.

Proof. Let $g \in \mathcal{H}, R \in \mathcal{R}^{N}, \mathbb{P}(R)=\left\{S_{-}, S_{+}, D_{-}, D_{+}\right\}$, and $x \equiv \varphi^{g}(R)$. Thus $p(R)$ induces $C \in \mathcal{C}$ and $C=S_{-} \cup D_{+}$. First note that, by definition, $z \geq z[C]$. Recall, that $D_{+}=\Gamma\left(S_{-}\right)$. That is, a supplier in $S_{-}$only has potential demanders in $D_{+}$. Note that $S_{+}=\Gamma\left(D_{-}\right)$. That is, a demander in $D_{-}$only has potential suppliers in $S_{+}$. Thus,

$$
z_{i}=z_{i}[C] \text { if } i \in S_{-} \cup D_{-} \quad \text { and } \quad z_{i} \geq z_{i}[C] \text { if } i \notin S_{+} \cup D_{+} .
$$

Let $i \in S_{-} \cup D_{-}$. If $p\left(R_{i}\right) \leq z_{i}[C]$, then by (a) in the definition of $g, x_{i}=p\left(R_{i}\right)$. And, if $p\left(R_{i}\right)>z_{i}[C]$, then by (b) in the definition of $g, x_{i} \geq z_{i}[C]$. Moreover, by 
Proposition 1, $\varphi^{g}(R) \in P(R)$. Thus, by the Efficiency Lemma, $x_{i} \leq p\left(R_{i}\right)$. Thus, $z_{i}[C] \leq x_{i} \leq p\left(R_{i}\right)$. Thus, by single-peakedness, $x_{i} R_{i} z_{i}[C]=z_{i}$. Similarly, for each $i \notin S_{-} \cup D_{-}, p\left(R_{i}\right) \leq x_{i} \leq z_{i}[C] \leq z_{i}$. Thus, by single-peakedness, $x_{i} R_{i} z_{i}$.

Next, we give a necessary and sufficient condition for an adjustment mechanism to satisfy the "voluntary trade" property used in BIMS' characterization of the egalitarian mechanism. Voluntary trade is the requirement that each agent finds what she gets at least as desirable as receiving nothing. If receiving a null assignment can be viewed as an outside option, it amounts to individual rationality.

Voluntary trade: For each $R \in \mathcal{R}^{N}$ and each $i \in N, \varphi_{i}(R) R_{i} 0$.

Corollary 2. A strategy-proof, efficient, and replacement-dominant mechanism $\varphi$ satisfies voluntary trade if and only if there is $g \in \mathcal{H}$ such that

$$
\text { for each } C \in \mathcal{C}, \quad q^{C}=0 \quad \text { and } \quad \varphi=\varphi^{g} \text {. }
$$

Proof. Let $\varphi$ denote a mechanism satisfying the above properties. By Theorem 1, there is $g \in \mathcal{H}$ such that $\varphi=\varphi^{g}$. Suppose $g$ does not satisfy (2): there is $C \in \mathcal{C}$ such that $q^{C} \neq 0$. Thus, there is $k \in S$ such that $q_{k}^{C}>0$. Let $R \in \mathcal{R}^{N}$ be such that $p\left(R_{i}\right)=X_{i}$ if $i$ is in $[C \cap S] \cup[D \backslash C]$ and $p\left(R_{i}\right)=0$ otherwise. Then, by conditions (a) and (b) in the definition of an adjustment function, $\varphi^{g}(R)=q^{C}$. If $k \in C \cap S$, since $\left.q^{C}\right|_{C} \in Z(C)$ and $q_{k}^{C}>0, \sum_{D \cap C} q_{l}^{C}=\sum_{S \cap C} q_{l}^{C}>0$. Thus, there is $j \in C \cap D$ such that $p\left(R_{j}\right)=0<q_{j}^{C}=\varphi_{j}^{g}(R)=\varphi(R)$. Then, by single-peakedness, $0 P_{j} \varphi_{j}(R)$. This contradicts the assumption that $\varphi$ satisfies voluntary trade. We obtain an analogous contradiction if $k \in S \backslash C$.

Conversely, if $g \in \mathcal{H}$ is such that (2) holds, Proposition 2 implies that $\varphi^{g}$ satisfies voluntary trade.

Corollary 2 illustrates how demanding voluntary trade is. An adjustment rule satisfying it cannot guarantee any agent a positive amount of the resource.

\subsubsection{The converse of Theorem 1}

We identify the adjustment mechanisms satisfying replacement-dominance. The following condition on $g \in \mathcal{H}$ must be added to (a),(b), and (c): 
(d) If $\tilde{p}$ induces $C$ as well, $\tilde{q}^{1} \equiv g\left(q^{C}, \tilde{p}_{i}, p_{-i}\right), \ldots, \tilde{q}^{n} \equiv g\left(\tilde{q}^{n-1}, \tilde{p}_{i}, p_{-i}\right)$, then, for each $H$ in $\{C, N \backslash C\}$ and each $I$ in $\{S, D\}$,

$$
i \in H \cap I, \quad \tilde{p}_{i} \geq p_{i} \Rightarrow\left\{\begin{array}{ccc}
q_{j}^{n} \geq \tilde{q}_{j}^{n} & \text { if } & j \in H \cap I \backslash\{i\} \\
q_{j}^{n} \leq \tilde{q}_{j}^{n} & \text { if } & j \in H \cap[N \backslash I] \\
q_{j}^{n}=\tilde{q}_{j}^{n} & \text { if } & j \in N \backslash H
\end{array} .\right.
$$

Let $\mathcal{G} \subseteq \mathcal{H}$ denote the class of adjustment functions satisfying (d). In the context of Sprumont's model, the class of adjustment mechanisms specified by a $g \in \mathcal{G}$ coincides with the class of mechanisms introduced by Barberà et al. (1997).

Proposition 3. If $g \in \mathcal{G}$, then $\varphi^{g}$ is strategy-proof, efficient, and replacementdominant.

Theorem 2. A mechanism $\varphi$ is strategy-proof, efficient, and replacement-dominant if and only if there is $g \in \mathcal{G}$ such that $\varphi=\varphi^{g}$.

\section{$6 \quad$ Examples and applications}

Though the analysis in this paper applies to situations where allocation cannot rely on price adjustments, this does not necessarily rule out monetary considerations. In the allocation of workloads, there is a cost or wage associated with allocating a unit of the workload to each agent though these costs are taken to be fixed parameters. These parameters are likely to be important in recommending an allocation: the mechanism designer may need to minimize her total wage expenditure conditional on the workloads being distributed efficiently. A consequence of our analysis is that she could simultaneously ensure incentive compatibility, efficiency, and minimize total expenditures. We formalize these considerations associating a numeric benefit/cost measure to each agent.

Here, we describe examples of adjustment mechanisms based on this idea. These mechanisms include the egalitarian mechanisms of BIM and BIMS. As we will see, the chosen distributional objective in BIM and BIMS, egalitarianism, can be implemented by assigning all agents the same benefit/cost measure. As in BIM and BIMS, we focus on the case where resources are perfectly divisible.

The numeric benefit/cost measure we associate with each agent is a concave/convex function. For each $i \in N$, let $\mathcal{F}_{\boldsymbol{i}}$ denote the class of strictly concave and continuous functions $f_{i}: A_{i} \rightarrow \mathbb{R}^{14}$ The following mechanisms are indexed or parameterized by a profile $f \in \mathcal{F}^{N}$.

\footnotetext{
${ }^{14}$ The mechanisms described here are related to the parametric mechanisms characterized by
} 
Separably concave mechanism of parameterization $f \in \mathcal{F}^{N}, \varphi^{f}: \quad$ For each $R \in \mathcal{R}^{N}, \varphi^{f}(R) \equiv \arg \max \left\{\sum_{N} f_{i}\left(x_{i}\right): x \in P(R), x \leq p(R)\right\}$.

By the Feasibility and Efficiency Lemmas, $\{x \in P(R): x \leq p(R)\}$ is a bounded polyhedron defined by linear inequalities. Thus, it is a compact and convex subset of $\mathbb{R}_{+}^{N}$. Moreover, since the objective in the optimization problem above is a strictly concave function, every separably concave mechanism is well defined.

BIMS' egalitarian mechanism is the separably concave mechanism parameterized by $f \in \mathcal{F}^{N}$ where, for each $i \in N, f_{i}\left(x_{i}\right)=-x_{i}^{2}$. To see this, recall that BIMS' egalitarian mechanism is defined as the Lorenz-dominant ${ }^{15}$ element in the subset of efficient allocations at which no agent is assigned more than her peak: for each $R \in \mathcal{R}^{N}$, the BIMS-egalitarian allocation is the Lorenz-dominant point in $\{x \in$ $P(R): x \leq p(R)\}$. This implies that the BIMS-egalitarian allocation maximizes $\sum_{N}-x_{i}^{2}$ over the polytope $\{x \in P(R): x \leq p(R)\}$ (Schmeidler, 1979).

To see that the separably concave mechanisms are indeed adjustment mechanisms we can specify their adjustment functions.

Proposition 4. For each $f \in \mathcal{F}^{N}$ the adjustment function corresponding to $\varphi^{f}$ is $g: Z \times A^{N} \rightarrow Z$ such that, if $p \in A^{N}$ induces $C$ (Section 5.1),

(i) $q^{C}$ is allocation assigning 0 to each agent;

(ii) and, if $q^{0} \equiv q^{C}$ and, for each $t \in\{1, \ldots, n\}, q^{t} \equiv g\left(q^{t-1}, p\right)$, where

$$
\begin{aligned}
q^{t}=\arg \max \sum_{N} f_{i}\left(x_{i}\right) & \text { such that } x_{C} \in Z(C), x_{N \backslash C} \in Z(N \backslash C) \text {, and } \\
& x_{i}=p_{i} \quad \text { if } i \in[C \cap S] \cup[D \backslash C] \text { and } q_{i}^{t-1} \geq p_{i} \\
& x_{i}=p_{i} \quad \text { if } i \notin[C \cap S] \cup[D \backslash C] \text { and } q_{i}^{t-1} \leq p_{i} \\
& x_{i} \geq q_{i}^{t-1} \text { if } i \in[C \cap S] \cup[D \backslash C] \text { and } q_{i}^{t-1}<p_{i} \\
& x_{i} \leq q_{i}^{t-1} \quad \text { if } i \notin[C \cap S] \cup[D \backslash C] \text { and } q_{i}^{t-1}>p_{i} .
\end{aligned}
$$

\section{Unilateral assignment}

The results derived for the bilateral assignment problems studied until now can be extended to unilateral assignment problems. Firstly, observe that unilateral assign-

Young (1987) in the context of bankruptcy problems. (See Thomson (2003) for a survey of bankruptcy problems.) They are also reminiscent of the class of "collectively rational mechanisms" in the axiomatic theory of bargaining (Lensberg, 1987).

${ }^{15}$ Let $d \in \mathbb{N}$ and $x, y \in \mathbb{R}_{+}^{d}$. Let $x^{*}$ denote the rearrangement of the coordinates of $x$ such that $x_{1}^{*} \leq x_{2}^{*} \leq \cdots \leq x_{d}^{*}$ and define $y^{*}$ analogously. Then, $x$ Lorenz-dominates $y$ if, for each $k \in\{1, \ldots, d\}, \sum_{i=1}^{k} x_{i}^{*} \geq \sum_{i=1}^{k} y_{i}^{*}$. 
ment problems can be viewed as special cases of bilateral problems: simply take the demanders' peaks as fixed amounts to be allocated among suppliers subject to the network constraints.

More formally, recall from Section 2 that in unilateral assignment problems each demander $i$ has a singleton assignment space $A_{i}=\left\{X_{i}\right\}$. To nest a unilateral problem in a bilateral one, it suffices to ensure that in the bilateral problem we recommend allocations whereby each demander $i$ receives exactly $X_{i}$. By the Feasibility Lemma, a necessary and sufficient condition for it to be possible to allocate the demand profile $X_{D}$ among the suppliers is that,

$$
\text { for each } I \subseteq D, \quad \sum_{I} X_{i} \leq \sum_{\Gamma(I)} X_{i} .
$$

Under this condition we can still use the Efficiency Lemma to describe efficient allocations in unilateral problems by fixing demander preferences so that, for each demander $i, p\left(R_{i}\right)=X_{i}$.

Corollary 3. Suppose that condition (3) is satisfied and let $\varphi$ denote a mechanism defined on the domain of preference profiles $R \in \mathcal{R}^{N}$ such that $p\left(R_{D}\right)=X_{D}$. Then $\varphi$ is strategy-proof, efficient, replacement-dominant, and ensures that each demander $i$ is assigned $X_{i}$ if and only if there is $g \in \mathcal{H}$ such that

$$
\text { for each } C \in \mathcal{C} \text { and each } i \in D, \quad q_{i}^{C}=X_{i} \quad \text { and } \quad \varphi=\varphi^{g} .
$$

The proof is analogous to that of Corollary 2 and is thus omitted. We now define the analogues of the separably concave mechanisms defined in the previous section for unilateral problems. ${ }^{16}$

Separably concave mechanism of parameterization $f \in \mathcal{F}^{S}, \varphi^{f}: \quad$ For each $R \in \mathcal{R}^{N}, \varphi^{f}(R) \equiv \arg \min \left\{\sum_{S} f_{i}\left(x_{i}\right): x \in P(R), x_{D}=p\left(R_{D}\right)\right\}$.

By the same argument in Section 6, BIM's egalitarian mechanism is the separably concave mechanism parametrized by $f \in \mathcal{F}^{S}$ where, for each $i \in N, f_{i}\left(x_{i}\right)=-x_{i}^{2}$.

\section{Appendix}

We now introduce a number of network flow tools that are used throughout this appendix. Let $\lambda \in A^{N}$ and construct the following network $G(\lambda)$ : add a source node

\footnotetext{
${ }^{16}$ These mechanisms are well defined by the same arguments in Section 6 .
} 
$\boldsymbol{s}$ and sink node $\boldsymbol{t}$ to the set of nodes in $G$ and define the arc set of $G(\lambda)$ is to be

$$
\mathcal{A} \equiv\{(i, j): i j \in G, i \in S, j \in D\} \cup\{(s, i): i \in S\} \cup\{(j, t): j \in D\} .
$$

Each arc $(i, j) \in \mathcal{A}$ has an arc capacity - an upper bound on the amount that can traverse through the arc $(i, j)$, from node $i$ to node $j$ - given by

$$
\boldsymbol{c}(\boldsymbol{i}, \boldsymbol{j}) \equiv \begin{cases}\lambda_{j} & \text { if } \quad j \in S \text { and } i=s \\ \infty & \text { if } \quad i j \in G, i \in S, j \in D \\ \lambda_{i} & \text { if } \quad i \in D \text { and } j=t\end{cases}
$$

An $s-\boldsymbol{t}$ flow in network $G(\lambda)$ specifies an amount traversing each arc in $G(\lambda)$, $\phi \in \mathbb{R}^{\mathcal{A}}$ such that,

(i) for each $(i, j) \in \mathcal{A}, 0 \leq \phi_{(i, j)} \leq c(i, j)$ (the flow through an arc does not exceed the arc's capacity);

(ii) for each $i \in S, \phi_{(s, i)}=\sum_{j \in D} \phi_{(i, j)}$, and, for each $i \in D, \phi_{(i, t)}=\sum_{j \in S} \phi_{(j, i)}$ (for each node other than $s$ or $t$ the amount entering that node is the same as the amount exiting it).

An $s-t$ flow $\boldsymbol{\phi}$ is maximal if $\sum_{i \in N} \phi_{(s, i)}$ is greater than that of any other $s-t$ flow. A cut in in network $G(\lambda)$ is a subset $K \subseteq N \cup\{s\}$ containing $s$. The capacity of a cut $K$ is given by $\sum_{i \in C, j \notin C} c(i, j)$. A min-cut is a minimum capacity cut.

Let $R \in \mathcal{R}^{N}$ and $p \equiv p(R)$. Consider network $G(p)$. By the max-flow min-cut theorem, the maximal $s-t$ flow is equal to the minimum capacity of a cut in network $G(p)$. Note that if $K$ is a min-cut we have

$$
K \cap D=\Gamma(K \cap S) .
$$

Otherwise, $K \cap D \nsubseteq \Gamma(K \cap S)$ and the cut has an infinite capacity. If $C$ is such that $[K \cap D] \backslash \Gamma(K \cap S) \neq \varnothing$, its capacity could be further reduced. In both cases $C$ is not a min-cut. By (4), if $K$ is a min-cut, its capacity is

$$
\sum_{i \in K, j \notin K} c(i, j)=\sum_{S \backslash K} p_{i}+\sum_{K \cap D} p_{i}=\sum_{S \backslash K} p_{i}+\sum_{\Gamma(K \cap S)} p_{i} .
$$

Note that the class of cuts minimizing (5) is closed under unions and intersections. Thus, there is a unique inclusion-minimal min-cut $K^{\mathbf{m i n}}$. This yields an equivalent description of partition $\mathbb{P}(R)$.

Lemma 3. Let $R \in \mathcal{R}^{N}$ and $\mathbb{P}(R)=\left\{S_{-}, S_{+}, D_{-}, D_{+}\right\}$. 
(i) $S_{-} \equiv K^{\min } \cap S, D_{+} \equiv K^{\min } \cap D, S_{+} \equiv S \backslash S_{-}$, and $D_{-} \equiv D \backslash D_{+}$.

(ii) If $p(R)$ induces $C$ (as defined Section 5.1), then $C=K^{\min } \backslash\{s\}$.

Proof. (i) Let $I=S \cap K$ and note that $\sum_{S \backslash K} p_{i}+\sum_{\Gamma(K \cap S)} p_{i}=\sum_{S} p_{i}-\left(\sum_{I} p_{i}-\right.$ $\left.\sum_{\Gamma(I)} p_{i}\right)$. Thus, $K^{\mathrm{min}}$ is the inclusion minimal min-cut in (5) if and only if $K^{\mathrm{min}} \cap S$ is the inclusion-minimal minimizer in (1). (ii) By definition, if $p(R)$ induces $C$, $C=S_{-} \cup D_{+}$. Thus (ii) follows from (i).

\section{A Proof of Lemma 1}

Proof of Lemma 1. Suppose that, for each $i \in N, X_{i}=\infty$ and let $N^{\prime}=N$. Then the Lemma reduces to showing that an allocation $x$ is feasible, $x \in Z$, if and only if, for each $I \subseteq S$ (similarly, if $I \subseteq D$ ), $\sum_{I} x_{i} \leq \sum_{\Gamma(I)} x_{j}$ and $\sum_{S} x_{i}=\sum_{D} x_{j}$. If $x \in Z$, then there is no $I \subseteq N$ such that $\sum_{I} x_{i}>\sum_{\Gamma(I)} x_{j}$ because the agents in $I$ can receive at most $\sum_{\Gamma(I)} x_{j}$. Conversely, suppose that $x \in \mathbb{R}_{+}^{N}$ is such that for each $I \subseteq N, \sum_{I} x_{i} \leq \sum_{\Gamma(I)} x_{j}$ and $\sum_{S} x_{i}=\sum_{D} x_{j}$. By the max-flow min-cut theorem, there is a maximal flow $\phi \in \mathbb{R}^{\mathcal{A}}$ in network $G(x)$ with value equal to the minimum capacity of a cut in the network. Since, for each $I \subseteq N, \sum_{I} x_{i} \leq \sum_{\Gamma(I)} x_{j}$, this cut is $\{s\}$ and its capacity is $\sum_{S} x_{i}=\sum_{i \in S} \phi_{(s, i)}$. Since $\sum_{S} x_{i}=\sum_{D} x_{j}$, matrix $\left(\phi_{(i, j)}: i \in S, j \in D\right)$ implements allocation $x$. Thus, $x \in Z$. A similar proof characterizes, for each $N^{\prime} \subseteq N, Z\left(N^{\prime}\right)$.

Suppose that there is $i \in N$ with $X_{i}<\infty$. Let $N^{\prime} \subseteq N, S^{\prime} \equiv S \cap N^{\prime}$, and $D^{\prime} \subseteq D \cap N^{\prime}$. If $x \in Z\left(N^{\prime}\right)$, then (i) and (ii) in Lemma 1 are immediate. Conversely, suppose that $x \in \mathbb{R}_{+}^{N^{\prime}}$ is such that (i)-(ii) hold. Then, for each $I \subseteq S^{\prime}$,

$$
\sum_{I} x_{i} \leq \min \left\{\sum_{\Gamma\left(K ; N^{\prime}\right)} x_{i}+\sum_{I \backslash K} X_{i}: K \subseteq I\right\} \leq \min \left\{\sum_{I} X_{i}, \sum_{\Gamma\left(I ; N^{\prime}\right)} x_{i}\right\} .
$$

Similarly, for each $J \subseteq S^{\prime}, \sum_{J} x_{i} \leq \sum_{J} X_{s}$ and $\sum_{J} x_{i} \leq \sum_{\Gamma\left(J ; N^{\prime}\right)} x_{i}$. Thus,

$$
\text { for each } I \subseteq B^{\prime}, \sum_{I} x_{i} \leq \sum_{\Gamma\left(I ; N^{\prime}\right)} x_{i} \text {, and, for each } J \subseteq S^{\prime}, \sum_{J} x_{i} \leq \sum_{\Gamma\left(J ; N^{\prime}\right)} x_{i} .
$$

We have already shown these conditions to be necessary and sufficient for the feasibility of $x$ in the absence of the upper bounds $\left(X_{i}\right)_{i \in N^{\prime}}$. For each $i \in S^{\prime}$, letting $I \equiv\{i\}$ in (6), $x_{i} \leq X_{i}$. Similarly, for each $i \in D^{\prime}, x_{i} \leq X_{i}$. 


\section{B Proof of Propositions 1 and 3}

The proof relies on the following Lemma.

Lemma 4. Let $R \in \mathcal{R}^{N}$ and $i \in N$. Let $R^{\prime} \in \mathcal{R}^{N}$ be such that, for each $j \in N \backslash\{i\}$, $R_{j}^{\prime}=R_{j}$. Let $p \equiv p(R)$ and $p^{\prime} \equiv p\left(R^{\prime}\right)$. Let $K$ and $K^{\prime}$ denote the inclusion-wise minimal min-cuts in $G(p)$ and $G\left(p^{\prime}\right)$, respectively.

(a) If $i \in S \cap K$ and $p_{i}^{\prime} \geq p_{i}, K^{\prime}=K$. If $i \in S \backslash K$ and $p_{i}^{\prime} \leq p_{i}, K^{\prime}=K$.

(b) If $i \in D \cap K$ and $p_{i}^{\prime} \leq p_{i}, K^{\prime}=K$. If $i \in D \backslash K$ and $p_{i}^{\prime} \geq p_{i}, K^{\prime}=K$.

(c) If $K^{\prime}=K$, then $\mathbb{P}(R)=\mathbb{P}\left(R^{\prime}\right)$.

Proof. By (4), the min-cut $K$ in $G(p)$ satisfies $K \cap D=\Gamma(K \cap S)$. Thus, its capacity in network $G(p)$ is

$$
\operatorname{cap}(K, \boldsymbol{p}) \equiv \sum_{S \backslash K} p_{k}+\sum_{K \cap D} p_{k}=\sum_{S \backslash K} p_{k}+\sum_{\Gamma(K \cap S)} p_{k} .
$$

Moreover, the min-cuts in $G(p)$ are precisely the minimizers of (7).

(a) Let $i \in S \cap K$ and $p_{i}^{\prime} \geq p_{i}$. Since the capacity of a cut in network $G(p)$ is no greater than its capacity in $G\left(p^{\prime}\right)$,

$$
\operatorname{cap}(K, p) \leq \operatorname{cap}\left(K^{\prime}, p\right) \leq \operatorname{cap}\left(K^{\prime}, p^{\prime}\right) \leq \operatorname{cap}\left(K, p^{\prime}\right)
$$

By (7), $i \in S \cap K$ implies that $\operatorname{cap}(K, p)=\operatorname{cap}\left(K, p^{\prime}\right) . \operatorname{Thus,} \operatorname{cap}\left(K^{\prime}, p^{\prime}\right)=\operatorname{cap}\left(K, p^{\prime}\right)$ and $K$ is a min-cut in $G\left(p^{\prime}\right)$. Since $K^{\prime}$ is the inclusion-wise minimal min-cut in $G\left(p^{\prime}\right)$, $K \supseteq K^{\prime}$. Likewise, $\operatorname{since} \operatorname{cap}(K, p)=\operatorname{cap}\left(K^{\prime}, p\right), K \subseteq K^{\prime}$. Thus, $K=K^{\prime}$.

Let $i \in S \backslash K$ and $p_{i}^{\prime} \leq p_{i}$. By (7),

$$
0 \leq p_{i}-p_{i}^{\prime}=\operatorname{cap}(K, p)-\operatorname{cap}\left(K, p^{\prime}\right)
$$

Similarly, the capacity of each cut in $G\left(p^{\prime}\right)$ not containing $i$ is $p_{i}-p_{i}^{\prime} \geq 0$ less than its capacity in $G(p)$ and the capacity of each cut in $G\left(p^{\prime}\right)$ containing $i$ is the same as in $G(p)$. Since $K$ is a min-cut in $G(p), \operatorname{cap}\left(K, p^{\prime}\right)=\operatorname{cap}\left(K^{\prime}, p^{\prime}\right)$. Since $K^{\prime}$ is inclusionminimal, $K^{\prime} \subseteq K$. Since $K$ is inclusion-minimal in $G(p)$ and $i \notin K, K^{\prime} \supseteq K$. Thus, $C=C^{\prime}$. The proof of (b) is symmetric. Condition (c) follows from Lemma 3.

Proof of Propositions 1 and 3. Let $g \in \mathcal{H}$. We first prove that $\varphi^{g}$ satisfies the properties in Proposition 1. Let $R \in \mathcal{R}^{N}, p \equiv p(R)$, and suppose that $p$ induces $C$. Let

$$
q^{1} \equiv g\left(q^{C}, p\right), \quad q^{2} \equiv g\left(q^{1}, p\right), \ldots, \quad q^{n} \equiv g\left(q^{n-1}, p\right) .
$$

Let $S_{-}, S_{+}, D_{-}, D_{+}$denote the cells of partition $\mathbb{P}(R)$. By Lemma $3, C=S_{-} \cup D_{+}$ and $N \backslash C=S_{+} \cup D_{-}$. 
$\boldsymbol{\varphi}^{\boldsymbol{g}}$ is efficient: We prove that $q^{n} \in P(R)$. By the definition of an adjustment function $g, q^{n}$ is in $Z\left(S_{-} \cup D_{+}\right) \times Z\left(S_{+} \cup D_{-}\right)$. Thus, by the Efficiency Lemma, if $q^{n} \notin P(R)$ there is $i \in S_{-} \cup D_{-}$such that $q_{i}^{n}>p_{i}$ or $i \in S_{+} \cup D_{+}$such that $q_{i}^{n}<p_{i}$. Note that $q^{n} \neq q^{n-1}$ : otherwise, by $(\mathrm{a}), q_{i}^{n}=p_{i}$. By (a) in the definition of adjustment function $g$, for each $j \in N \backslash\{i\}, q_{j}^{n}=p_{j}{ }^{17}$ By Lemma 3, since $\{s\} \cup S_{-} \cup D_{+}$is a min-cut in $G(p)$ and $D_{+}=\Gamma\left(S_{-}\right), \sum_{S_{-}} p_{k} \geq \sum_{D_{+}} p_{k}$. Thus, if $i \in S_{-}$,

$$
\sum_{S_{-}} q_{k}^{n}>\sum_{S_{-}} p_{k} \geq \sum_{D_{+}} p_{k}=\sum_{D_{+}} q_{k}^{n} .
$$

Then, $q_{S_{-} \cup D_{+}}^{n} \notin Z\left(S_{-} \cup D_{+}\right)$, a contradiction. We derive analogous contradictions if $i \in D_{-}, i \in S_{+}$, or $i \in D_{+}$. Thus, for each $i \in S_{-} \cup D_{-}, q_{i}^{n} \leq p_{i}$, and, for each, $i \in S_{+} \cup D_{+}, q_{i}^{n} \geq p_{i}$. By the Efficiency Lemma, $q^{n} \in P(R)$.

$\boldsymbol{\varphi}^{\boldsymbol{g}}$ is strategy-proof: Let $i \in N$ and $R^{\prime} \in \mathcal{R}^{N}$ be such that, for each $j \in N \backslash\{i\}$, $R_{j}^{\prime}=R_{j}$. Let $S_{-}^{\prime}, S_{+}^{\prime}, D_{-}^{\prime}, D_{+}^{\prime}$ denote the cells of partition $\mathbb{P}\left(R^{\prime}\right)$. Let $p^{\prime} \equiv p\left(R^{\prime}\right)$ and $x^{\prime} \equiv \varphi^{g}\left(R^{\prime}\right)$. We prove that $q_{i}^{n} R_{i} x_{i}^{\prime}$. If $q_{i}^{n}=p_{i}$ we are done, so assume otherwise. Throughout, we use the facts that $q^{n} \in P(R)$ and $x^{\prime} \in P\left(R^{\prime}\right)$. We distinguish two cases:

Case 1: $\mathbb{P}(R)=\mathbb{P}\left(R^{\prime}\right)$. Suppose that $i \in S_{-}$. Since $\varphi^{g}$ is efficient, by the Efficiency Lemma, $q_{i}^{n}<p\left(R_{i}\right)$. By (b) and (c) in the definition of an adjustment function, $q_{i}^{n} \neq x_{i}^{\prime}$ requires there is $t \in\{1, \ldots, n\}$ such that $q_{i}^{t} \geq p_{i}^{\prime}$. Then, by (a) in the definition of an adjustment function, $x_{i}^{\prime}=p_{i}^{\prime}$. Thus, $x_{i}^{\prime} \leq q_{i}^{t} \leq q_{i}^{n}=x_{i}<p_{i}$. By single-peakedness, $q_{i}^{n} R_{i} x_{i}^{\prime}$. Analogously, if $i \in D_{-}, i \in S_{+}$, or $i \in D_{+}$we arrive at the same conclusion.

Case 2: $\mathbb{P}(R) \neq \mathbb{P}\left(R^{\prime}\right)$. Suppose that $i \in S_{-}$. By Lemma 4, $p_{i}>p_{i}^{\prime}$ or else $\mathbb{P}(R)=\mathbb{P}\left(R^{\prime}\right)$. Moreover, $i \in S_{+}^{\prime} \cdot{ }^{18}$ Let $K \equiv S_{-} \cap S_{+}^{\prime}$ and $L \equiv D_{-}^{\prime} \cap D_{+}$. By the definition of $\mathbb{P}(R), K \subseteq S_{-}$implies $\Gamma(K) \subseteq D_{+}$. By the Efficiency Lemma, $x_{S_{+}^{\prime} \cup D_{-}^{\prime}}^{\prime} \in Z\left(S_{+}^{\prime} \cup D_{-}^{\prime}\right)$. Thus, all supply from $K \cap \Gamma(L)$ is received by demanders in

\footnotetext{
${ }^{17}$ An adjustment occurs, i.e. $q^{t} \neq q^{t}-1$, only if there is $l \in N$ such that $p_{l} \neq q_{l}^{t-1}$ and $p_{l}=q_{l}^{t}$. Thus, if $n-1$ adjustments have taken place at least $n-1$ agents are receiving their peaks.

${ }^{18}$ Let $K$ and $K^{\prime}$ are the inclusion-minimal min-cuts in $G(p)$ and $G\left(p^{\prime}\right)$ respectively. By Lemma 3 , $S_{-}=K \cap S$ so $i \notin S \backslash K$. Recalling the definition in $(7), \operatorname{cap}\left(K^{\prime}, p^{\prime}\right)=\operatorname{cap}\left(K^{\prime}, p\right) \geq \operatorname{cap}(K, p) \geq$ $\operatorname{cap}\left(K, p^{\prime}\right) \geq \operatorname{cap}\left(K^{\prime}, p^{\prime}\right)$. Thus $K$ is a min-cut in $G\left(p^{\prime}\right)$ and $K^{\prime}$ is a min-cut in $G(p)$. Since both are inclusion-minimal, $K=K^{\prime}$. By Lemma 3 , this would yield $\mathbb{P}(R)=\mathbb{P}\left(R^{\prime}\right)$, counter to our assumption.
} 
$L$. We thus obtain the second inequality below: ${ }^{19}$

$$
x_{i}^{\prime}+\sum_{[K \cap \Gamma(L)] \backslash\{i\}} p_{k}^{\prime} \leq \sum_{K \cap \Gamma(L)} x_{k}^{\prime} \leq \sum_{L} x_{l}^{\prime} \leq \sum_{L} p_{k}^{\prime} .
$$

Similarly, by the definition of $\mathbb{P}\left(R^{\prime}\right), \Gamma(L) \subseteq S_{+}^{\prime}$. By the Efficiency Lemma, $q_{S_{-} \cup D_{+}} \in$ $Z\left(S_{-} \cup D_{+}\right)$. Thus, the demanders in $L$ can receive resources solely from suppliers in $K$. Thus,

$$
q_{i}^{n}+\sum_{[K \cap \Gamma(L)] \backslash\{i\}} p_{k} \geq \sum_{K \cap \Gamma(L)} q_{k}^{n} \geq \sum_{L} q_{l}^{n} \geq \sum_{L} p_{k} .
$$

Since $p_{-i}=p_{-i}^{\prime}$, combining (8) and (9) yields $p_{i} \geq q_{i}^{n} \geq x_{i}^{\prime}$. By single-peakedness, $q_{i}^{n} R_{i} x_{i}^{\prime}$, as desired.

Next, suppose that $R^{\prime}$ is such that $\mathbb{P}(R) \neq \mathbb{P}\left(R^{\prime}\right)$ and $i \in S_{+}$. By Lemma 4, $p_{i}<p_{i}^{\prime}$ and $i \in S_{-}^{\prime}$. Let $K \equiv S_{-}^{\prime} \cap S_{+}$and $L \equiv D_{-} \cap D_{+}^{\prime}$. By analogous arguments to those above we arrive at $x_{i}^{\prime} \geq q_{i}^{n} \geq p_{i}$. By single-peakedness, $q_{i}^{n} R_{i} x_{i}^{\prime}$ again, as desired. The cases where $i \in D_{-}$and $i \in D_{+}$are symmetric.

$\varphi^{\boldsymbol{g}}$ is replacement-dominant: To prove Proposition 3 assume that $g$ is in $\mathcal{G}$, not just in $\mathcal{H}$. Let $i \in N$ and $\tilde{R} \in \mathcal{R}^{N}$ be such that, for each $j \in N \backslash\{i\}, R_{j}=\tilde{R}_{j}$. Assume, as in the hypothesis of replacement-dominance, that $\mathbb{P}(\tilde{R})=\mathbb{P}(R)$. By Lemma 3, $p(R)$ and $p(\tilde{R})$ induce $C$. Thus, $\varphi(\tilde{R}) \equiv \tilde{q}^{n}$ where, $\tilde{q}^{0} \equiv q^{C}$ and

$$
\tilde{q}^{1} \equiv g\left(\tilde{q}^{0}, p(\tilde{R})\right), \quad \tilde{q}^{2} \equiv g\left(\tilde{q}^{1}, p(\tilde{R})\right), \ldots, \quad \tilde{q}^{n} \equiv g\left(\tilde{q}^{n-1}, p(\tilde{R})\right) .
$$

Let $i \in S_{+}$. Then, by $(\mathrm{d}), p\left(\tilde{R}_{i}\right) \leq p\left(R_{i}\right)$ implies:

[for each $\left.k \in S_{+} \backslash\{i\}, p\left(R_{k}\right) \leq q_{k}^{n} \leq \tilde{q}_{k}^{n}\right] \Rightarrow\left[\right.$ for each $\left.k \in S_{+} \backslash\{i\}, q_{k}^{n} R_{k} \tilde{q}_{k}^{n}\right]$,

[for each $\left.k \in D_{-}, p\left(R_{k}\right) \geq q_{k}^{n} \geq \tilde{q}_{k}^{n}\right] \Rightarrow\left[\right.$ for each $k \in D_{-}, q_{k}^{n} R_{k} \tilde{q}_{k}^{n}$,

[for each $\left.k \in N \backslash\left[S_{+} \cup D_{-}\right], q_{k}^{n}=\tilde{q}_{k}^{n}\right] \Rightarrow\left[\right.$ for each $\left.S_{-} \cup D_{+}, q_{k}^{n} R_{k} \tilde{q}_{k}^{n}\right]$.

Altogether, for each $k \in N \backslash\{i\}, q_{k}^{n} R_{k} \tilde{q}_{k}^{n}$. Similarly, $p\left(\tilde{R}_{i}\right) \geq p\left(R_{i}\right)$ implies, for each $k \in N \backslash\{i\}, \tilde{q}_{k}^{n} R_{k} q_{k}^{n}$. The cases $i \in S_{-}, i \in D_{-}$, and $i \in D_{+}$are analogous.

\section{Proof of Theorems 1 and 2}

\section{C.1 Preliminaries}

Lemma 5. Let $\varphi$ be a strategy-proof, efficient, and replacement-dominant mechanism. Let $R \in \mathcal{R}^{N}$ and let $S_{-}, S_{+}, D_{-}, D_{+}$be the cells of partition $\mathbb{P}(R)$. Let $I \subseteq N$

\footnotetext{
${ }^{19}$ The other inequalities follow from the Efficiency Lemma because $K \subseteq S_{+}^{\prime}$ and $L \subseteq D_{-}^{\prime}$.
} 
and $R^{\prime} \in \mathcal{R}^{N}$ be such that, for each $j \in N \backslash I, R_{j}^{\prime}=R_{j}$.

(i) Let $(K, L) \in\left\{\left(S_{-}, D_{+}\right),\left(D_{-}, S_{+}\right)\right\}$. If $I \subseteq K$ and $p\left(R_{I}^{\prime}\right) \geq p\left(R_{I}\right)$ then,

$$
\begin{aligned}
k \in K \backslash I & \Rightarrow \varphi_{k}(R) \geq \varphi_{k}\left(R^{\prime}\right), \\
k \in L & \Rightarrow \varphi_{k}(R) \leq \varphi_{k}\left(R^{\prime}\right), \\
k \in N \backslash[K \cup L] & \Rightarrow \varphi_{k}(R)=\varphi_{k}\left(R^{\prime}\right), \\
i \in I, \quad \varphi_{i}\left(R_{i}^{\prime}, R_{-i}\right)=\varphi_{i}(R) & \Rightarrow \varphi\left(R_{i}^{\prime}, R_{-i}\right)=\varphi(R) .
\end{aligned}
$$

If $I \subseteq L$ and $p\left(R_{I}^{\prime}\right) \leq p\left(R_{I}\right)$ then,

$$
\begin{aligned}
k \in L \backslash I & \Rightarrow \varphi_{k}(R) \leq \varphi_{k}\left(R^{\prime}\right), \\
k \in K & \Rightarrow \varphi_{k}(R) \geq \varphi_{k}\left(R^{\prime}\right), \\
k \in N \backslash[K \cup L] & \Rightarrow \varphi_{k}(R)=\varphi_{k}\left(R^{\prime}\right) \\
i \in I, \quad \varphi_{i}\left(R_{i}^{\prime}, R_{-i}\right)=\varphi_{i}(R) & \Rightarrow \varphi\left(R_{i}^{\prime}, R_{-i}\right)=\varphi(R) .
\end{aligned}
$$

(ii) If $I \subseteq S_{-} \cup D_{-}$and $p\left(R_{I}^{\prime}\right) \geq p\left(R_{I}\right)$ then,

$$
\begin{aligned}
k \in\left[S_{-} \cup D_{-}\right] \backslash I & \Rightarrow \varphi_{k}(R) \geq \varphi_{k}\left(R^{\prime}\right), \\
k \in S_{+} \cup D_{+} & \Rightarrow \varphi_{k}(R) \leq \varphi_{k}\left(R^{\prime}\right) .
\end{aligned}
$$

If $I \subseteq S_{+} \cup D_{+}$and $p\left(R_{I}^{\prime}\right) \leq p\left(R_{I}\right)$ then,

$$
\begin{aligned}
k \in S_{-} \cup D_{-} & \Rightarrow \varphi_{k}(R) \leq \varphi_{k}\left(R^{\prime}\right), \\
k \in\left[S_{+} \cup D_{+}\right] \backslash I & \Rightarrow \varphi_{k}(R) \geq \varphi_{k}\left(R^{\prime}\right) .
\end{aligned}
$$

Proof. Let $p \equiv p(R), p^{\prime} \equiv p\left(R^{\prime}\right), x \equiv \varphi(R)$ and $x^{\prime} \equiv \varphi\left(R^{\prime}\right)$. Let the remaining notation be the same as in the statement of the Lemma.

(i) Suppose first that $I \equiv\{i\}$ and $p_{i}^{\prime}>p_{i}$. By Lemma 4, the inclusion minimal min-cuts in $G(p)$ and $G\left(p^{\prime}\right)$ are the same. Thus, $\mathbb{P}(R)=\mathbb{P}\left(R^{\prime}\right)$. That is, the hypothesis of replacement-domiance is satisfied and this axiom has bite in evaluating changes in preferences.

Since $\mathbb{P}(R)=\mathbb{P}\left(R^{\prime}\right)$ and $i \in S_{-} \cup D_{-}$, by the Efficiency Lemma, $x_{i}^{\prime} \leq p_{i}^{\prime}$. By strategy-proofness, $x_{i} \leq x_{i}^{\prime} \leq p_{i}^{\prime}$. By replacement-domiance, either (a) [for each $j \in N \backslash\{i\}, x_{j} R_{j} x_{j}^{\prime}$ ] or (b) [for each $j \in N \backslash\{i\}, x_{j}^{\prime} R_{j} x_{j}$.

Case 1: $x_{i}^{\prime}=x_{i}$. Suppose that (a) holds. By the Efficiency Lemma, for each $k \in\left[S_{-} \cup D_{-}\right] \backslash\{i\}, x_{k}^{\prime} \leq p_{k}^{\prime}=p_{k}$ and, for each $k \in D_{+} \cup S_{+}, x_{k}^{\prime} \geq p_{k}^{\prime}=p_{k}$. Thus, $x_{k} R_{k} x_{k}^{\prime}$ implies $x_{k}^{\prime} \leq x_{k}$ and, for each $k \in S_{+} \cup D_{+}, x_{k}^{\prime} \geq x_{k}$. By the Efficiency 
Lemma, $\sum_{S_{-}} x_{k}^{\prime}=\sum_{D_{+}} x_{k}^{\prime}$ and $\sum_{S_{+}} x_{k}^{\prime}=\sum_{D_{-}} x_{k}^{\prime}$. Thus, $x=x^{\prime}$. When (b) holds, a similar argument yields $x=x^{\prime}$. This confirms (i) when $I \equiv\{i\} \subseteq K$.

Case 2: $x_{i}^{\prime}>x_{i}$. Suppose $i \in K=S_{-}$. By the Efficiency Lemma, $\sum_{S_{-}} x_{k}^{\prime}=$ $\sum_{D_{+}} x_{k}^{\prime}$. Thus, by feasibility,

[there is $j \in S_{-} \backslash\{i\}$ such that $x_{j}^{\prime}<x_{j}$ ] or [there is $j \in D_{+}$such that $x_{j}^{\prime}>x_{j}$ ].

Suppose the former holds. By the Efficiency Lemma, since $\mathbb{P}(R)=\mathbb{P}\left(R^{\prime}\right), x_{j}^{\prime}<$ $x_{j} \leq p_{j}^{\prime}=p_{j}$. By single-peakedness, $x_{j} P_{j} x_{j}^{\prime}$. By replacement-domiance, for each $k \in N \backslash\{i\}, x_{k} R_{k} x_{k}^{\prime}$. Thus, by the Efficiency Lemma and single-peakedness,

$$
k \in K \backslash\{i\} \Rightarrow x_{k}^{\prime} \leq x_{k} \quad \text { and } \quad k \in L \Rightarrow x_{k}^{\prime} \geq x_{k}
$$

Suppose, instead that [there is $j \in D_{+}$such that $x_{j}^{\prime}>x_{j}$. By the Efficiency Lemma, since $\mathbb{P}(R)=\mathbb{P}\left(R^{\prime}\right), x_{j}^{\prime}>x_{j} \geq p\left(R_{j}\right)$. Thus, $x_{j} P_{j} x_{j}^{\prime}$. Thus, by replacementdomiance, for each $k \in N \backslash\{i\}, x_{k} R_{k} x_{k}^{\prime}$. By the Efficiency Lemma and singlepeakedness, we reach the same conclusion as in (10). Symmetrically, we arrive at the same statement when $K=D_{-}$.

We have shown that if $x_{i}^{\prime}>x_{i}$ then, for each $k \in N \backslash\{i\}, x_{k} R_{k} x_{k}^{\prime}$. Since $\mathbb{P}(R)=\mathbb{P}\left(R^{\prime}\right)$, the Efficiency Lemma implies that, for each $k \in S_{+} \cup D_{+}, x_{k}^{\prime} \geq$ $x_{k} \geq p_{i}$ and, for each $k \in S_{-} \cup D_{-}, x_{k}^{\prime} \leq x_{k} \leq p_{k}$. Since, $\sum_{S_{-}} x_{k}^{\prime}=\sum_{D_{+}} x_{k}^{\prime}$ and $\sum_{S_{+}} x_{k}^{\prime}=\sum_{D_{-}} x_{k}^{\prime}$

$$
k \in N \backslash(K \cup L)=D_{-} \cup S_{+} \Rightarrow x_{k}=x_{k}^{\prime} .
$$

Combining (10) and (11), confirms the first statement in (i) when $\{i\}=I \subseteq K$. Now, suppose that $|I|>1$, say $I \equiv\{1, \ldots, k\}$. By Lemma $4, \mathbb{P}(R)=\mathbb{P}\left(R_{1}^{\prime}, R_{-1}\right)=$ $\mathbb{P}\left(R_{\{1,2\}}^{\prime}, R_{N \backslash\{1,2\}}\right)=\cdots=\mathbb{P}\left(R^{\prime}\right)$. We can thus repeat the argument for $|I|=1(|I|-$ times) and arrive at the first statement in (i). A fully analogous argument establishes the second statement in (i), where $I \subseteq L$.

(ii) Let $I \subseteq S_{-} \cup D_{-}$. Let $S^{\prime} \equiv I \cap S_{-}$and $D^{\prime} \equiv I \cap D_{-}$. The first statement in (ii) now follow by applying (i) first to $S^{\prime}$ and then to $D^{\prime}$. Likewise, the case where $I \subseteq S_{+} \cup D_{+}$follows from (i).

Next, we show that our axioms imply two technically useful properties.

Peaks-only: For each $\left\{R, R^{\prime}\right\} \subseteq \mathcal{R}^{N}, p(R)=p\left(R^{\prime}\right)$ implies $\varphi(R)=\varphi\left(R^{\prime}\right)$. 
Uncompromisingness: For each $R \in \mathcal{R}^{N}$, each $i \in N$, and each $R_{i}^{\prime} \in \mathcal{R}_{i}$,

$$
\begin{aligned}
& {\left[p\left(R_{i}\right)<\varphi_{i}(R) \text { and } p\left(R_{i}^{\prime}\right) \leq \varphi_{i}(R)\right]} \\
& {\left[p\left(R_{i}\right)>\varphi_{i}(R) \text { and } p\left(R_{i}^{\prime}\right) \geq \varphi_{i}(R)\right]}
\end{aligned} \Rightarrow \varphi_{i}\left(R_{i}^{\prime}, R_{-i}\right)=\varphi_{i}(R) \text {. }
$$

Lemma 6. A strategy-proof, efficient, and replacement-dominant mechanism is peaks-only and uncompromising.

Proof. Let $\varphi$ be a strategy-proof, efficient, and replacement-dominant mechanism. Let $\left\{R, R^{\prime}\right\} \subseteq \mathcal{R}^{N}$ be such that $p(R)=p\left(R^{\prime}\right)$. Since $G(p(R))=G\left(p\left(R^{\prime}\right)\right), \mathbb{P}(R)=$ $\mathbb{P}\left(R^{\prime}\right)$. Let $i \in N$. By strategy-proofness and efficiency, $\varphi_{i}\left(R_{i}^{\prime}, R_{-i}\right)=\varphi_{i}(R)$. By Lemma 5 (i), $\varphi\left(R_{i}^{\prime}, R_{-i}\right)=\varphi(R)$. Repeating this argument $n-1$ more times we find that $\varphi(R)=\varphi\left(R^{\prime}\right)$. This establishes peaks-only.

Let $R \in \mathcal{R}^{N}, i \in N$, and $R_{i}^{\prime} \in \mathcal{R}_{i}$ be such that $\left[p\left(R_{i}\right)>\varphi_{i}(R)\right.$ and $\left.p\left(R_{i}^{\prime}\right) \geq \varphi_{i}(R)\right]$. Let $x \equiv \varphi(R)$ and $x^{\prime} \equiv \varphi\left(R_{i}^{\prime}, R_{-i}\right)$. If $x_{i}<x_{i}^{\prime}$, let $\hat{R}_{i} \in \mathcal{R}_{i}$ be such that $p\left(\hat{R}_{i}\right)=p\left(R_{i}\right)$ and $x_{i}^{\prime} \hat{P}_{i} x_{i}$. By peaks-only, $x_{i}=\varphi_{i}\left(\hat{R}_{i}, R_{-i}\right)$. Thus, $\varphi_{i}\left(R_{i}^{\prime}, R_{-i}\right) \hat{P}_{i} \varphi_{i}\left(\hat{R}_{i}, R_{-i}\right)$, a violation of strategy-proofness. If $x_{i}>x_{i}^{\prime}$, a similar argument yields an analogous contradiction. Thus, $x_{i}^{\prime}=x_{i}$. This establishes uncompromisingness.

\section{C.2 Proof of Theorems 1 and 2}

By Proposition 1, if $g \in \mathcal{H}, \varphi^{g}$ is strategy-proof and efficient. By Proposition 3, if $g \in \mathcal{G} \subseteq \mathcal{H}$, then $\varphi^{g}$ is also replacement-dominant.

Conversely, let $\varphi$ denote a strategy-proof, efficient, and replacement-dominant mechanism. We prove that there is a $g \in \mathcal{H}$ such that $\varphi=\varphi^{g}$. (Lemma 5 establishes condition (d) in Section 5.2.2.)

Notation: If $R, R^{t}, \tilde{R}, \tilde{R}^{t}, \cdots \in \mathcal{R}^{N}$, we let $p \equiv p(R), \tilde{p} \equiv p(\tilde{R}), \tilde{p}^{t} \equiv p\left(\tilde{R}^{t}\right)$, and so forth. For each $I \subseteq N$, let $\boldsymbol{r}[\boldsymbol{I}] \equiv\left(r_{i}[I]\right)_{i \in N}$ where, for each $i \in N, r_{i}[I]$ is $X_{i}$ if $i \in[I \cap S] \cup[D \backslash I]$ and 0 otherwise. For each $I \subseteq N$, let $\mathbf{R}[I] \in \mathcal{R}^{N}$ be such that $p(\mathbf{R}[C])=r[C]$.

By Lemma 6, $\varphi$ satisfies peaks-only and uncompromisingness. We will use these facts throughout without further reference to the Lemma. We now define our candidate adjustment function: let $g: Z \times A^{N} \rightarrow Z$ be such that, for each $p \in A^{N}$,

(i) if $p$ induces $C$, let $q^{C} \equiv \varphi(\mathbf{R}[C])$,

(ii) and, if $q^{0}=q^{C}$, for each $t \in\{1, \ldots, n\}$, let $g\left(q^{t-1}, p\right) \equiv \varphi\left(R^{t}\right)$ where 


$$
R^{t} \in \mathcal{R}^{N} \text { is s.t. } p_{i}^{t}=\left\{\begin{array}{cllll}
p_{i} & \text { if } & i \in[C \cap S] \cup[D \backslash C] & \text { and } & q_{i}^{t-1} \geq p_{i} \\
X_{i} & \text { if } & i \in[C \cap S] \cup[D \backslash C] & \text { and } & q_{i}^{t-1}<p_{i} \\
p_{i} & \text { if } & i \notin[C \cap S] \cup[D \backslash C] & \text { and } & q_{i}^{t-1} \leq p_{i} \\
0 & \text { if } & i \notin[C \cap S] \cup[D \backslash C] & \text { and } & q_{i}^{t-1}>p_{i} .
\end{array}\right.
$$

This specification of $g$ is meaningful because $\varphi$ is peaks-only.

Step 1. If $p \in A^{N}$ induces $C$, then $q^{C}$ is as defined in Section 5.1.

Proof. Suppose that $p$ induces $C$. By (i) and (ii) in Lemma 3 and Lemma $4, r[C]$ induces $C$ as well. Thus, by (i) and (ii) in Lemma 3 if $S_{-}, S_{+}, D_{-}, D_{+}$denote the cells of partition $\mathbb{P}(\mathbf{R}[C]), C=S_{-} \cup D_{+}$. Since $\varphi$ is efficient, $q^{C} \equiv \varphi(\mathbf{R}[C]) \in P(\mathbf{R}[C])$. Thus, by the Efficiency Lemma and Lemma $3, q^{C} \in Z(C) \times Z(N \backslash C)$.

Step 2. If $p \in A^{N}$ induces $C$ and $q^{0}=q^{C}$, then, for each $t \in\{1, \ldots, n\}$,

$$
\left.g\left(q^{t-1}, p\right)\right|_{C} \in Z(C) \text { and }\left.g\left(q^{t-1}, p\right)\right|_{N \backslash C} \in Z(N \backslash C) .
$$

Proof. Let $p, C$, and $q^{0}, q^{1}, \ldots, q^{n}$ be as introduced in the statement. For each $t \in\{1, \ldots, n\}$, let $R^{t}, p^{t}$ be defined with respect to $p$ and $q^{t-1}$ as in (12). Let $t \in\{1, \ldots, n\}$. Note that, for each $i \in[C \cap S] \cup[D \backslash C], p_{i}^{t} \geq p_{i}$ and, for each $i \notin[C \cap S] \cup[D \backslash C], p_{i}^{t} \leq p_{i}$. Let $N \equiv\left\{i_{1}, \ldots, i_{n}\right\}$. By Lemma 3, $\{s\} \cup C$ is the inclusion minimal min-cut in $G(p)$. By Lemma $4,\{s\} \cup C$ is the inclusion minimal min-cut across the networks

$$
G(p), G\left(p_{i_{1}}^{t}, p_{N \backslash\left\{i_{1}\right\}}\right), G\left(p_{\left\{i_{1}, i_{2}\right\}}^{t}, p_{N \backslash\left\{i_{1}, i_{2}\right\}}\right), \ldots, G\left(p^{t}\right) .
$$

Thus, by Lemma 3 , for each $R \in \mathcal{R}^{N}$ with $p=p(R), \mathbb{P}(R)=\mathbb{P}\left(R_{i_{1}}^{t}, R_{N \backslash\left\{i_{1}\right\}}\right)=\cdots=$ $\mathbb{P}\left(R^{t}\right)$. Recall that, by Lemma 3 , if $\mathbb{P}(R) \equiv\left\{S_{-}, S_{+}, D_{-}, D_{+}\right\}$, then $C=S_{-} \cup D_{+}$. By efficiency, $\varphi\left(R^{t}\right) \in P\left(R^{t}\right)$ and, by definition, $g\left(q^{t-1}, p\right)=\varphi\left(R^{t}\right)$. Thus, by the Efficiency Lemma, $\left.g\left(q^{t-1}, p\right)\right|_{C} \in Z(C)$ and $\left.g\left(q^{t-1}, p\right)\right|_{N \backslash C} \in Z(N \backslash C)$.

The following step establishes that $\varphi$ coincides with $\varphi^{g}$. We then establish (Step 4) that $g$ satisfies properties (a), (b), and (c) in the definition of an adjustment function.

Step 3. Let $R \in \mathcal{R}^{N}$, suppose that $p \equiv p(R)$ induces $C$, and let

$$
q^{0} \equiv q^{C}, \quad q^{1} \equiv g\left(q^{0}, p\right), \ldots, \quad q^{n} \equiv g\left(q^{n-1}, p\right) .
$$

Then, $\varphi(R)=g\left(q^{n-1}, p\right)$. 
Proof. Let $R, p, C$, and $q^{0}, q^{1}, \ldots, q^{n}$ be as defined in the statement. Recall that, by Lemma 3 , if $\mathbb{P}(R) \equiv\left\{S_{-}, S_{+}, D_{-}, D_{+}\right\}, C=S_{-} \cup D_{+}$and $N \backslash C=S_{+} \cup D_{-}$. Let $N_{-} \equiv S_{-} \cup D_{-}$and $N_{+} \equiv S_{+} \cup D_{+}$. For each $t \in\{1, \ldots, n\}$, let

$N_{-}^{t} \equiv\left\{k \in N_{-}: p_{k}<q_{k}^{t-1}\right\}, \quad N_{+}^{t} \equiv\left\{k \in N_{+}: p_{k}>q_{k}^{t-1}\right\}, \quad N_{0}^{t} \equiv\left\{k \in N: p_{k}=q_{k}^{t-1}\right\}$.

Claim 1. Let $t \in\{1, \ldots, n\}$.

$$
\begin{aligned}
& \text { 1. } N_{-}^{t} \cup N_{+}^{t}=\varnothing \Rightarrow \varphi(R)=q^{t}=q^{t-1} \text { and } \\
& \text { 2. } i \in N_{0}^{t} \cup N_{-}^{t} \cup N_{+}^{t} \Rightarrow \varphi_{i}(R)=q_{i}^{t}=p_{i} .
\end{aligned}
$$

We now prove the Claim. For each $t \in\{1, \ldots, n\}$, let $R^{t}, p^{t}$ be defined with respect to $p$ and $q^{t-1}$ as in (12). Note that, by our definition of $g$, letting $R^{0} \equiv \mathbf{R}[C]$, for each $t \in\{1, \ldots, n\}, g\left(q^{t-1}, p\right)=\varphi\left(R^{t}\right)$. Thus, for each $t \in\{0,1, \ldots, n\}, q^{t}=\varphi\left(R^{t}\right)$.

1. Let $t \in\{1, \ldots, n\}$ be such that $N_{-}^{t} \cup N_{+}^{t}=\varnothing$. Note that, for each $i \in N_{-}$, $p_{i} \leq p_{i}^{t-1}$ and, for each $i \in N_{+}, p_{i} \geq p_{i}^{t-1}$. Thus,

$$
\begin{array}{rlr}
i \in N_{-} \Rightarrow q_{i}^{t-1}=\varphi_{i}\left(R^{t-1}\right) & =\varphi_{i}\left(R_{i}, R_{-i}^{t-1}\right) & \text { by uncompromisingness, } \\
& \leq \varphi_{i}\left(R_{N_{-}}, R_{N_{+}}^{t-1}\right) & \text { by Lemma } 5 \text { (ii), } \\
& \leq \varphi_{i}(R) & \text { by Lemma } 5 \text { (ii); } \\
i \in N_{+} \Rightarrow q_{i}^{t-1}=\varphi_{i}\left(R^{t-1}\right) & =\varphi_{i}\left(R_{i}, R_{-i}^{t-1}\right) & \text { by uncompromisingness, } \\
& \geq \varphi_{i}\left(R_{N_{-}}^{t-1}, R_{N_{+}}\right) & \text {by Lemma } 5 \text { (ii), } \\
& \geq \varphi_{i}(R) & \text { by Lemma } 5 \text { (ii). }
\end{array}
$$

By Step 2, $\sum_{S_{-}} q_{i}^{t-1}=\sum_{D_{+}} q_{i}^{t-1}$. By the Efficiency Lemma, $\sum_{S_{-}} \varphi_{i}(R)=\sum_{D_{+}} \varphi_{i}(R)$. Thus, by Step 2 and the above inequalities,

$$
\sum_{S_{-}} \varphi_{i}(R) \geq \sum_{S_{-}} q_{i}^{t-1}=\sum_{D_{+}} q_{i}^{t-1} \geq \sum_{D_{+}} \varphi_{i}(R)
$$

Thus, $\left.\varphi(R)\right|_{S_{-} \cup D_{+}}=q_{S_{-} \cup D_{+}}^{t-1}$. Likewise, $\left.\varphi(R)\right|_{S_{+} \cup D_{-}}=q_{S_{+} \cup D_{-}}^{t-1}$. Thus, $\varphi(R)=$ $q^{t-1}$ and it remains to show that $q^{t-1}=q^{t}$. Let $N \backslash N_{0}^{t} \equiv\left\{i_{1}, i_{2}, \ldots, i_{k}\right\}$. By uncompromisingness,

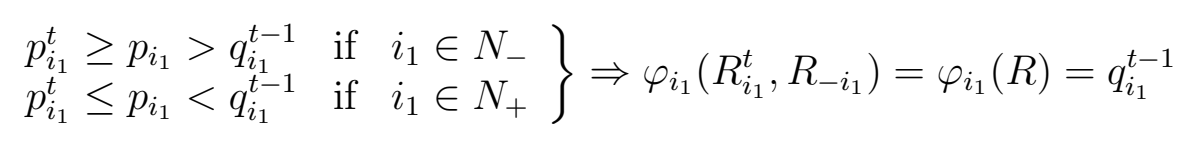


By Lemma 5 (i), for each $j \in N \backslash\left\{i_{1}\right\}, \varphi_{j}\left(R_{i_{1}}^{1}, R_{-i_{1}}\right)=\varphi_{j}(R)=q_{j}^{t-1}$. Likewise,

$$
\varphi_{i_{2}}\left(R_{\left\{i_{1}, i_{2}\right\}}^{t}, R_{N \backslash\left\{i_{1}, i_{2}\right\}}\right)=\varphi_{i_{2}}(R)
$$

and, for each $j \in N \backslash\left\{i_{2}\right\}, \varphi_{j}\left(R_{\left\{i_{1}, i_{2}\right\}}^{t}, R_{N \backslash\left\{i_{1}, i_{2}\right\}}\right)=\varphi_{j}(R)$,

$$
\varphi_{i_{k}}\left(R_{\left\{i_{1}, \ldots, i_{k}\right\}}^{t}, R_{N \backslash\left\{i_{1}, \ldots, i_{k}\right\}}\right)=\varphi_{i_{k}}(R),
$$

and, for each $j \in N \backslash\left\{i_{k}\right\}, \varphi_{j}\left(R_{\left\{i_{1}, \ldots, i_{k}\right\}}^{t}, R_{N \backslash\left\{i_{1}, \ldots, i_{k}\right\}}\right)=\varphi_{j}(R)$.

Since $R^{t} \equiv\left(R_{\left\{i_{1}, \ldots, i_{k}\right\}}^{t}, R_{N \backslash\left\{i_{1}, \ldots, i_{k}\right\}}\right), \varphi(R)=\varphi\left(R^{t}\right) \equiv g\left(q^{t-1}, p\right)$. Thus, $q^{t}=q^{t-1}$.

2. Let $t \in\{1, \ldots, n\}$ be such that $N_{-}^{t} \cup N_{+}^{t} \neq \varnothing$. By the Efficiency Lemma,

$$
\begin{aligned}
i \in N_{0}^{t} \cap N_{-} & \Rightarrow \varphi_{i}\left(R_{i}, R_{-i}^{t-1}\right) \leq p_{i}=q_{i}^{t-1}=\varphi_{i}\left(R^{t-1}\right), \\
i \in N_{0}^{t} \cap N_{+} & \Rightarrow \varphi_{i}\left(R_{i}, R_{-i}^{t-1}\right) \geq p_{i}=q_{i}^{t-1}=\varphi_{i}\left(R^{t-1}\right), \\
i \in N_{-}^{t} & \Rightarrow \varphi_{i}\left(R_{i}, R_{-i}^{t-1}\right) \leq p_{i}<q_{i}^{t-1}=\varphi_{i}\left(R^{t-1}\right), \\
i \in N_{+}^{t} & \Rightarrow \varphi_{i}\left(R_{i}, R_{-i}^{t-1}\right) \geq p_{i}>q_{i}^{t-1}=\varphi_{i}\left(R^{t-1}\right) .
\end{aligned}
$$

From (13) and strategy-proofness,

$$
i \in N_{0}^{t} \cap N_{-} \text {or } i \in N_{0}^{t} \cap N_{+} \Rightarrow \varphi_{i}\left(R_{i}, R_{-i}^{t-1}\right)=p_{i},
$$

Suppose $i \in N_{-}^{t}$ and $\varphi_{i}\left(R_{i}, R_{-i}^{t-1}\right)<p_{i}$. Let $\tilde{R}_{i} \in \mathcal{R}_{i}$ be such that

$$
q_{i}^{t-1} \tilde{P}_{i} \varphi_{i}\left(R_{i}, R_{-i}^{t-1}\right) \quad \text { and } \quad \tilde{p}_{i}=p_{i}
$$

By peaks-only, $\varphi_{i}\left(R_{i}, R_{-i}^{t-1}\right)=\varphi_{i}\left(\tilde{R}_{i}, R_{-i}^{t-1}\right)$. Thus, $q_{i}^{t-1} \equiv \varphi_{i}\left(R_{i}^{t-1}, R_{-i}^{t-1}\right) \tilde{P}_{i} \varphi_{i}\left(\tilde{R}_{i}, R_{-i}^{t-1}\right)$, contradicting strategy-proofness. Analogously, if $i \in N_{+}^{t}, \varphi_{i}\left(R_{i}, R_{-i}^{t-1}\right)=R_{i}$. Thus,

$$
i \in N_{-}^{t} \cup N_{+}^{t} \Rightarrow \varphi_{i}\left(R_{i}, R_{-i}^{t-1}\right)=p_{i} .
$$

Again, note that $p_{N_{-}} \leq p_{N_{-}}^{t-1}$ and $p_{N_{+}} \geq p_{N_{+}}^{t-1}$. Thus, by the above observations,

$$
\begin{aligned}
i \in N_{-}^{t} \cup\left[N_{0}^{t} \cap N_{-}\right] \Rightarrow p_{i}=\varphi_{i}\left(R_{i}, R_{-i}^{t-1}\right) & \leq \varphi_{i}\left(R_{N_{-}}, R_{N_{+}}^{t-1}\right) & & \text { by Lemma } 5 \text { (ii), } \\
& \leq \varphi_{i}(R) & & \text { by Lemma } 5 \text { (ii), } \\
i \in N_{+}^{t} \cup\left[N_{0}^{t} \cap N_{+}\right] \Rightarrow p_{i}=\varphi_{i}\left(R_{i}, R_{-i}^{t-1}\right) & \geq \varphi_{i}\left(R_{N_{-}}^{t-1}, R_{N_{+}}\right) & & \text {by Lemma } 5 \text { (ii), } \\
& & & \\
& & & \text { by Lemma } 5 \text { (ii) }
\end{aligned}
$$


By the Efficiency Lemma, $\left.\varphi(R)\right|_{N_{-}} \leq p_{N_{-}}$and $\left.\varphi(R)\right|_{N_{+}} \geq p_{N_{+}}$. Thus, (14) implies

$$
i \in N_{0}^{t} \cup N_{-}^{t} \cup N_{+}^{t} \Rightarrow \varphi_{i}(R)=p_{i} .
$$

Next, we show that, for each $i \in N_{0}^{t} \cup N_{-}^{t} \cup N_{+}^{t}, \varphi_{i}(R)=\varphi_{i}\left(R^{t}\right)$. By (15) and (14),

$$
i \in N_{-}^{t} \cup\left[N_{0}^{t} \cap N_{-}\right] \text {or } i \in N_{+}^{t} \cup\left[N_{0}^{t} \cap N_{+}\right] \Rightarrow \varphi_{i}(R)=\varphi_{i}\left(R_{i}, R_{-i}^{t-1}\right) .
$$

Let $N_{-}^{t} \cup\left[N_{0}^{t} \cap N_{-}\right] \equiv\left\{i_{1}, i_{2}, \ldots, i_{k}\right\}$ and $N_{+}^{t} \cup\left[N_{0}^{t} \cap N_{+}\right] \equiv\left\{i_{k+1}, i_{k+2}, \ldots, i_{\ell}\right\}$. Then,

$$
\begin{aligned}
& \varphi_{i_{1}}(R)=\varphi_{i_{1}}\left(R_{i_{1}}, R_{-i}^{t-1}\right) \\
& \leq \varphi_{i_{1}}\left(R_{\left\{i_{1}, i_{2}\right\}}, R_{N_{-} \backslash\left\{i_{1}, i_{2}\right\}}^{t-1}, R_{N_{+}}^{t-1}\right) \quad \text { by Lemma } 5 \text { (ii), } \\
& \vdots \\
& \leq \varphi_{i_{1}}\left(R_{\left\{i_{1}, i_{2}, \ldots, i_{k}\right\}}, R_{N_{-} \backslash\left\{i_{1}, i_{2}, \ldots, i_{k}\right\}}^{t-1}, R_{N_{+}}^{t-1}\right) \quad \text { by Lemma } 5 \text { (ii), } \\
& \leq \varphi_{i_{1}}\left(R_{\left\{i_{1}, i_{2}, \ldots, i_{k+1}\right\}}, R_{N_{-} \backslash\left\{i_{1}, i_{2}, \ldots, i_{k}\right\}}^{t-1}, R_{N_{+} \backslash\left\{i_{k+1}\right\}}^{t-1}\right) \quad \text { by Lemma } 5 \text { (ii), } \\
& \leq \varphi_{i_{1}}\left(R_{\left\{i_{1}, i_{2}, \ldots, i_{k+2}\right\}}, R_{N_{-} \backslash\left\{i_{1}, i_{2}, \ldots, i_{k}\right\}}^{t-1}, R_{N_{+} \backslash\left\{i_{k+1}, i_{k+2}\right\}}^{t-1}\right) \quad \text { by Lemma } 5 \text { (ii), } \\
& \vdots \\
& \leq \varphi_{i_{1}}\left(R_{\left\{i_{1}, i_{2}, \ldots, i_{\ell}\right\}}, R_{N_{-} \backslash\left\{i_{1}, i_{2}, \ldots, i_{k}\right\}}^{t-1}, R_{N_{+} \backslash\left\{i_{k+1}, \ldots, i_{\ell}\right\}}^{t-1}\right) \quad \text { by Lemma } 5 \text { (ii), } \\
& \leq p_{i_{1}} \quad \text { by the Efficiency Lemma, } \\
& =\varphi_{i_{1}}(R)
\end{aligned}
$$

Note that $R^{t} \equiv\left(R_{\left\{i_{1}, i_{2}, \ldots, i_{\ell}\right\}}, R_{N \backslash\left\{i_{1}, i_{2}, \ldots, i_{\ell}\right\}}^{t-1}\right)$. Thus, $\varphi_{i_{1}}\left(R^{t}\right)=p_{i_{1}}=\varphi_{i_{1}}(R)$. Since the indexing $\left\{i_{1}, i_{2}, \ldots, i_{k}\right\}$ is arbitrary,

$$
i \in N_{-}^{t} \cup\left[N_{0}^{t} \cap N_{-}\right] \Rightarrow \varphi_{i}\left(R^{t}\right)=\varphi_{i}(R)=p_{i} .
$$

A symmetric argument establishes the same condition for each $i \in N_{+}^{t} \cup\left[N_{0}^{t} \cap N_{+}\right]$. Altogether,

$$
i \in N_{0}^{t} \cup N_{-}^{t} \cup N_{+}^{t} \Rightarrow \varphi_{i}(R)=\varphi_{i}\left(R^{t}\right)
$$

Step 4. g satisfies properties (a), (b), and (c) in Section 5.1.

Proof. Let $p \in A^{N}$ and suppose that $p$ induces $C$. Let $q^{0} \equiv q^{C}, q^{1} \equiv g\left(q^{0}, p\right), \ldots, q^{n} \equiv$ $g\left(q^{n-1}, p\right)$. For each $t \in\{1, \ldots, n\}$, let $R^{t}, p^{t}$ be defined with respect to $p$ and $q^{t-1}$ as in (12). By (i) and (ii) in Lemma 3, there is a unique Gallai-Edmonds partition of $N$ corresponding to $C$. Let $S_{-}, S_{+}, D_{-}, D_{+}$denote this partition's cells so that $S_{-} \cup D_{-}$ coincides with $[S \cap C] \cup[D \backslash C]$ and $S_{+} \cup D_{+}$coincides with the complement. 
(a) This condition is equivalent to Claim 1 established in the proof of Step 3.

From our definition of $g$, letting $R^{0} \equiv \mathbf{R}[C]$,

$$
q^{C}=\varphi\left(R^{0}\right) \text { and, for each } t \in\{1, \ldots, n\}, g\left(q^{t-1}, p\right) \equiv \varphi\left(R^{t}\right)
$$

(b) Let $t \in\{1, \ldots, n\}$.

Let $i \in S_{-} \cup D_{-}$be such that $q_{i}^{t-1}<p_{i}$. Then, by (12), $p_{i}^{t}=p_{i}^{t-1}$. If $j \in N \backslash\{i\}$ is such that $p_{j}^{t} \neq p_{j}^{t-1}$,

$$
j \in S_{-} \cup D_{-} \Rightarrow p_{j}^{t}<p_{j}^{t-1} \text { and } j \in S_{+} \cup D_{+} \Rightarrow p_{j}^{t}>p_{j}^{t-1} \text {. }
$$

Thus, by Lemma 5 (ii), $\varphi_{i}\left(R^{t}\right) \geq \varphi_{i}\left(R^{t-1}\right)$. Thus, by (16), $q_{i}^{t} \geq q_{i}^{t-1}$.

Let $i \in S_{+} \cup D_{+}$be such that $q_{i}^{t-1}>p_{i}$. Analogously, $q_{i}^{t} \leq q_{i}^{t-1}$.

(c) Let $i \in N$ and $\tilde{p} \in A^{N}$ be such that, for each $j \in N \backslash\{i\}, \tilde{p}_{j}=p_{j}$.

Let $i \in S_{-} \cup D_{-}$be such that $q_{i}^{0}<p_{i} \leq \tilde{p}_{i}$. By Lemmas 3 and $4, \tilde{p}$ induces $C$ as well. Let

$$
\tilde{q}^{0} \equiv q^{C}, \quad \tilde{q}^{1} \equiv g\left(\tilde{q}^{0}, \tilde{p}\right), \ldots, \quad \tilde{q}^{n} \equiv g\left(\tilde{q}^{n-1}, \tilde{p}\right) .
$$

For each $t \in\{1, \ldots, n\}$, let $\tilde{R}^{t}, \tilde{p}^{t}$ be defined with respect to $\tilde{p}$ and $\tilde{q}^{t-1}$ as in (12). Since $\tilde{q}_{i}^{0}=q_{i}^{0}<p_{i} \leq \tilde{p}_{i}, R^{1}=\tilde{R}^{1}$. Thus, by uncompromisingness, $\varphi\left(R^{1}\right)=\varphi\left(\tilde{R}^{1}\right)$. By $(16), q^{1}=\tilde{q}^{1}$. Likewise, inductively, for each $t \in\{1, \ldots, n\}$, such that $q_{i}^{t-1}<p_{i} \leq \tilde{p}_{i}, q^{t}=\tilde{q}^{t}$.

Let $i \notin S_{-} \cup D_{-}$be such that $q_{i}^{0}>p_{i} \geq \tilde{p}_{i}$. Analogously, we find that, for each $t \in\{1, \ldots, n\}$, such that $q_{i}^{t-1}>p_{i} \geq \tilde{p}_{i}, q^{t}=\tilde{q}^{t}$.

\section{References}

Barberà, S., M. Jackson, and A. Neme (1997). Strategy-proof allotment rules. Games and Economic Behavior 18(1), $1-21$.

Bochet, O., R. İlkılıç, and H. Moulin (2013). Egalitarianism under earmark constraints. Journal of Economic Theory 148, 535-562.

Bochet, O., R. İlkılıç, H. Moulin, and J. Sethuraman (2012). Balancing supply and demand under bilateral constraints. Theoretical Economics 7, 395-423.

Bogomolnaia, A. and H. Moulin (2004). Random matching under dichotomous preferences. Econometrica $72(1), 257-279$. 
Gale, D. (1957). A theorem on flows in networks. Pacific Journal of Mathematics 7(1), 1073-1082.

Kıbrıs, O. and S. Küçükşenel (2009). Uniform trade rules for uncleared markets. Social Choice and Welfare 32, 101-121.

Korte, B. and J. Vygen (2001). Combinatorial Optimiazation: Theory and Algorithms. Springer.

Lensberg, T. (1987). Stability and collective rationality. Econometrica 55, 935-961.

Massó, J. and A. Neme (2007). Bribe-proof rules in the division problem. Games and Economic Behavior 61, 331-343.

Moulin, H. (1987). The pure compensation problem: Egalitarianism versus laissezfairism. Quarterly Journal of Economics 102, 769-783.

Satterthwaite, M. and H. Sonnenschein (1981). Strategy-proof allocation mechanisms at differentiable points. Review of Economic Studies 48(4), 587-597.

Schmeidler, D. (1979). A bibliographical note on a theorem by Hardy, Littlewood, and Polya. Journal of Economic Theory 59(20), 125-128.

Sprumont, Y. (1991). The division problem with single-peaked preferences. Econometrica 59(2), 509-519.

Thomson, W. (1997). The replacement principle in economies with single-peaked preferences. Journal of Economic Theory 76(1), 145 - 168.

Thomson, W. (1999). Welfare-domination and preference-replacement: a survey and open questions. Social Choice and Welfare 16, 373 - 394.

Thomson, W. (2003). Axiomatic and game-theoretic analysis of bankruptcy and taxation problems: a survey. Mathematical Social Siences 45(3), 249 - 297.

Thomson, W. (2007). Fair allocation rules. In K. Arrow, A. Sen, and K. Suzumura (Eds.), Handbook of Social Choice and Welfare, Volume 2, pp. 393-506. Elsevier.

Young, P. (1987). On dividing an amount according to claims or liabilities. Mathematics of Operations Research 12(3), $397-414$. 